\title{
Hierarchy of consistent n-component Cahn-Hilliard systems
}

\author{
Franck Boyer
}

Aix Marseille Université, CNRS, Centrale Marseille, I2M, UMR 7373, 13453 Marseille, France franck.boyer@univ-amu.fr

Sebastian Minjeaud

Univ. Nice Sophia Antipolis, CNRS, LJAD, UMR 7351, 06100 Nice, France Inria, Sophia Antipolis Méditerranée Research Centre, Project CASTOR minjeaud@unice.fr

\begin{abstract}
In this paper, we propose a new generalization of the well-known Cahn-Hilliard two-phase model for the modelling of n-phase mixtures. The model is derived using the consistency principle: we require that our n-phase model exactly coincide with the classical two-phase model when only two phases are present in the system. We give conditions for the model to be well-posed. We also present numerical results (including simulations obtained when coupling the Cahn-Hilliard system with the Navier-Stokes so as to obtain a phase-field model for multiphase flows) to illustre the capability of such modelling.
\end{abstract}

Keywords: Multicomponent diffuse interface model; Cahn-Hilliard system.

AMS Subject Classification: 35B35, 35K55, 76T30

\section{Introduction}

\subsection{General framework and main objectives}

The Cahn-Hilliard model (CH) was originally proposed (see Refs. 7,8 ) to describe spontaneous phase separation in a binary alloy below the critical temperature $\theta_{c}$. The starting point of the derivation of the Cahn-Hilliard equation consists in introducing a free energy $\mathcal{F}$, called Ginzburg-Landau free energy, of the form

$$
\mathcal{F}(c)=\int_{\Omega} \frac{A}{2}|\nabla c(x)|^{2}+B f(c(x)) d x, \quad A, B>0,
$$

where $\Omega \subset \mathbb{R}^{d}, d=1,2,3$ is a smooth bounded connected domain, $c$ stands for the order parameter (that is the atoms density of one of the (two) phases constituting the mixture) and $f: \mathbb{R} \rightarrow \mathbb{R}$ is a "coarse-grain" free energy: it is a double-well potential whose two wells characterize the pure phases of the mixture. More precisely, in this paper, we assume that

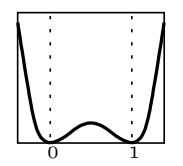

$$
\left\{\begin{array}{l}
f \text { is of class } \mathcal{C}^{1}(\mathbb{R}) \\
f \text { is symmetric: } f(c)=f(1-c) \text { for any } c \in \mathbb{R}, \\
f(0)=f(1)=0 \text { and } f>0 \text { elsewhere. }
\end{array}\right.
$$


Those assumptions imply in particular that $f^{\prime}(0)=f^{\prime}(1)=f^{\prime}(1 / 2)=0$ and that 0 and 1 are the unique minimisers of $f$ representing each of the two phases in the binary mixture. A thermodynamically consistent potential has the following expression

$$
f(c)=2 \theta_{c} c(1-c)+\theta(c \ln (c)+(1-c) \ln (1-c)), \quad 0<\theta<\theta_{c},
$$

but this singular potential do not satisfy the assumptions above and is very often replaced by a quartic polynomial approximation (after normalisation), of the following form

$$
f(c)=c^{2}(1-c)^{2}
$$

The coefficients $A$ and $B$ defining the free energy $\mathcal{F}$ can be connected with the two physically and numerically relevant quantities: the surface tension $\sigma$ between the two phases and the thickness $\varepsilon$ of the diffuse interface, through the following relations whose derivation is recalled in Appendix A

$$
A=\sigma \varepsilon a, \quad B=\frac{\sigma}{\varepsilon} b,
$$

where $a$ and $b$ are constants (depending only on the shape of the potential $f$ )

$$
a=\frac{\max _{[0,1]} \sqrt{f}}{\int_{0}^{1} \sqrt{f}}, \quad b=\frac{1}{2\left(\max _{[0,1]} \sqrt{f}\right) \int_{0}^{1} \sqrt{f}} .
$$

In the case of the fourth-order polynomial potential (1.3), we find $a=\frac{3}{2}, \quad b=12$.

Thus, once the potential shape $f$ is fixed, the free energy that we classically consider for a two-phase system with surface tension $\sigma$ and interface thickness $\varepsilon$, is given by

$$
\mathcal{F}_{\varepsilon}^{\sigma}(c)=\int_{\Omega} \frac{\sigma \varepsilon a}{2}|\nabla c|^{2}+\frac{\sigma}{\varepsilon} b f(c) d x .
$$

The evolution equation is then obtained by writting the mass balance

$$
\partial_{t} c+\operatorname{div}(J)=0,
$$

where $J$ is the mass flux related to the chemical potential $\mu$ by the constitutive equation

$$
J=-M \nabla \mu, \quad M>0,
$$

which is postulated so as to fulfill the main principles of thermodynamics. The chemical potential is usually defined as the functional derivative of the free energy with respect to the extensive parameter $c$

$$
\mu=-A \Delta c+B f^{\prime}(c)
$$

We eventually obtain the Cahn-Hilliard equation

$$
\left\{\begin{aligned}
\partial_{t} c & =\operatorname{div}(M \nabla \mu) \\
\mu & =-A \Delta c+B f^{\prime}(c) .
\end{aligned}\right.
$$


A different and somehow more rigorous derivation (based on additional microspic balance law) of this system is available in Ref. 19 (see also Ref. 29).

The Cahn-Hilliard system, with the quartic potential term (and, more generally, with a regular (nonsingular) potential $f$ ) is now well understood, at least from a mathematical point of view. In particular, one has a rather complete picture of existence, uniqueness and regularity results. Many authors have also analyzed the asymptotic properties of this dynamical system: existence of finite-dimensional attractors, convergence of single trajectories to steady states, for instance. Overviews of these theoretical results can be found in Refs. 10, 11, 31.

In this article, we deal with the generalization of the $\mathrm{CH}$ equation to multicomponent systems. Such extensions goes back to Refs. 9, 20, 30.

In order to properly derive the model we shall consider that the $n$-tuple $\mathbf{c}=$ $\left(c_{1}, \ldots, c_{n}\right)$ of order parameters belongs to a space $\mathscr{C}(\Omega)$ of smooth enough functions defined from $\Omega$ into $\mathbb{R}^{n}$, that we do not want to specify yet since the derivation of the model is made at the formal level. In practice, the mathematical analysis of the system will be performed in suitable Sobolev spaces, typically in $\mathscr{C}(\Omega)=\left(H^{1}(\Omega)\right)^{n}$, see Section 2.5.3.

As in the two-phase situation, the starting point of the derivation consists in considering a free energy $\mathcal{F}$ depending on the concentrations $\mathbf{c}$ of the $n$ phases in the system and on their gradients as follows

$$
\mathcal{F}(\mathbf{c})=\int_{\Omega} \overline{\mathcal{F}}(\mathbf{c}(x), \nabla \mathbf{c}(x)) d x,
$$

where $\overline{\mathcal{F}}: \mathbb{R}^{n} \times\left(\mathbb{R}^{d}\right)^{n} \mapsto \mathbb{R}$ is the free energy density.

The gradient system naturally associated with this free energy is of the following form

$$
\partial_{t} c_{i}+\operatorname{div}\left(\boldsymbol{J}_{i}\right)=0, \quad i=1, \ldots, n,
$$

where $\boldsymbol{J}_{i}$ stands for the total diffusion flux of the phase $i$. Classically, we assume that this total diffusion flux can be decomposed as follows

$$
\boldsymbol{J}_{i}=\sum_{j \neq i} \boldsymbol{J}_{i \rightarrow j}
$$

where the diffusion fluxes $\boldsymbol{J}_{i \rightarrow j}$ are assumed to be conservative and proportional to the differences between the gradients of generalized chemical potentials, that is

$$
\boldsymbol{J}_{i \rightarrow j}=-\boldsymbol{J}_{j \rightarrow i}, \quad \text { and } \quad \boldsymbol{J}_{i \rightarrow j}=\alpha_{i j} \nabla\left(\mu_{j}-\mu_{i}\right) .
$$

This particular form accounts for the Onsager reciprocity relations (see Refs. 26, $32,33)$. Note that the two constitutive assumptions (1.7) above imply that

$$
\alpha_{i j}=\alpha_{j i}, \quad \forall i \neq j .
$$

The chemical potential $\mu_{i}$ is defined as the functional derivatives of the total energy with respect to the order parameter $c_{i}$

$$
\mu_{i}=\frac{\delta \mathcal{F}}{\delta c_{i}} .
$$


Finally, the system reads

$$
\left\{\begin{aligned}
\frac{\partial c_{i}}{\partial t} & =-\operatorname{div}\left(\sum_{j=1}^{n} \alpha_{i j} \nabla \mu_{j}\right), \forall i=1, \ldots, n, \\
\mu_{i} & =\frac{\partial \mathcal{F}}{\partial c_{i}}, \quad \forall i=1, \ldots, n
\end{aligned}\right.
$$

where we have set for simplicity $\alpha_{i i}=-\sum_{j \neq i} \alpha_{i j}$, so that we have

$$
\sum_{j=1}^{n} \alpha_{i j}=0, \text { for any } i \in\{1, \ldots, n\} \text {. }
$$

We will also assume that

$$
\forall i \in\{1, \ldots, n\}, \exists j \in\{1, \ldots, n\}, \quad \alpha_{i j} \neq 0 .
$$

This assumption is natural since, if it does not hold for some $i$, we can deduce that the equation satisfied by $c_{i}$ in $(\mathrm{CH}[\mathcal{F}, \boldsymbol{\alpha}])$ reduces to $\frac{\partial c_{i}}{\partial t}=0$, which means that the phase number $i$ does not vary along time and thus can be simply removed from the system under study.

A derivation of such multi-component model based on microscopic balance law was proposed in Ref. 29. Several theoretical studies of such models are given in, e.g., Refs. 12, 13, 14, 15, 16, 17, 18, 28.

In this article, the goal is to provide an explicit expression of the parameters of the $n$-phase system (that is the density free energy $\overline{\mathcal{F}}$ and the coefficients $\alpha_{i j}$ ) from the two-phase "coarse grain" potential $f$, the interface width $\varepsilon$ and the surface tensions $\sigma_{i j}$ between each couple of distinct phases $(i, j)$ among the $n$ available phases.

Our approach is based on the consistency property: the $n$-phase model has to exactly coincide with the underlying two-phase models when only two phases are present (see Section 2.1 for a precise definition). Hence, we ensure by construction that a single set of PDEs is able to account for $n(n-1) / 2$ different two-phase situations in the system; we say that the model is consistent with the two-phase models. The importance of this last property when using such models in real multiphase modelling was mentionned in the numerical tests in Refs. 18, 24, where the authors observe that the third phase may appear during two-phase simulations using a three-phase model. However, the consistency property was first introduced and formalized in Ref. 3 and used to derive a suitable three-phase model. The model derived in Ref. 3 was based on the introduction of the (opposite of the) so-called spreading coefficients $\Sigma_{i}$ defined by

$$
\frac{1}{2}\left(\Sigma_{i}+\Sigma_{j}\right)=\sigma_{i j}, \quad \forall i, j .
$$

These relations uniquely define the coefficients $\Sigma_{i}(1 \leqslant i \leqslant n)$ when $n=3$, but unfortunately, as noted in Refs. $3,23,25$, this phase specific decomposition generates 
an over-determined system when $n \geqslant 4$ and can not be used. That is one of the reasons why the derivation of consistent models has to be revisited when $n \geqslant 4$.

Furthermore, when $n \geqslant 4$, we can require stronger consistency properties (see Section 2.1): the consistency with the three-phase models (that is the $n$-phase model may exactly coincide with the underlying three-phase models when only three phases are present), and so on. The ultimate purpose is to build a hierarchy of models such that the $n$-phase model is consistent with the $(n-1)$-phase models.

More precisely, our results are the following ones:

- we completely solve the problem for any choice of a symmetric double wellshaped smooth function $f$ (see assumption (1.2)) in the case where all the surface tensions (between each pair of phases) are the same.

- In the general situation of different surface tension coefficients, we provide $n$-phase models consistent with two-phase ones for any choice of $f$. To go further, we then restrict the study to the case of the usual quartic two-phase potential (1.3) (even though a similar analysis can be performed for higher order polynomial potentials), and provide $n$-phase models consistent with three-phase ones. Solving completely the problem still remains an open issue (see Section 3.2).

\subsection{Outline}

The article is organized as follows. In the end of the introduction, we recall some important properties of multi-component diffuse interface models which are already ensured thanks to the chosen general form of the model presented above. In Section 2, we then present the first steps of the derivation of the model by defining precisely the consistency properties we want to ensure and by providing the general form of the hierarchy of models. In Section 3, we detail the construction of the $n$-phase "coarse-grain" part of the energy in the different cases mentionned above. Finally, in Section 4, we provide some (preliminary) numerical experiments to illustrate the capabilities of such modelling in particular for phase-field simulations of multiphase flows.

\subsection{Notation and elementary properties}

Assuming that no mass transfer occurs at the boundary of the domain, that is $\boldsymbol{J}_{i \rightarrow j} \cdot \boldsymbol{n}=0$ on $\partial \Omega$ for any $i$ and $j$, or equivalently $\nabla \mu_{i} \cdot \boldsymbol{n}=0$ for any $i$, the formal energy estimate for this system reads

$$
\frac{d}{d t} \mathcal{F}(\mathbf{c})=\sum_{i=1}^{n} \int_{\Omega} \frac{\partial c_{i}}{\partial t} \frac{\delta \mathcal{F}}{\delta c_{i}} d x=\sum_{1 \leqslant i, j \leqslant n} \int_{\Omega} \alpha_{i j} \nabla \mu_{i} \cdot \nabla \mu_{j} d x .
$$

Moreover, the total volume conservation of each constituent holds

$$
\frac{d}{d t} \int_{\Omega} c_{i}(t, x) d x=0
$$


Since the order parameters $\left(c_{i}\right)_{i}$ are supposed to represent the concentrations of each phase in the mixture, the physically relevant solutions for the model should satisfy

$$
\sum_{i=1}^{n} c_{i}(t, x)=1, \text { for any } x \in \Omega, \text { for any } t>0 .
$$

Hence, it is natural to introduce the following functional space

$$
\mathscr{C}_{\mathcal{S}}(\Omega)=\{\mathbf{c} \in \mathscr{C}(\Omega), \quad \mathbf{c}(x) \in \mathcal{S}, \forall x \in \Omega\}, \text { with } \mathcal{S}=\left\{\mathbf{c} \in \mathbb{R}^{n}, \sum_{i=1}^{n} c_{i}=1\right\},
$$

and to only consider the following class of solutions, called perfect mixture solutions.

Definition 1.1. Any solution of System $((\mathrm{CH}[\mathcal{F}, \boldsymbol{\alpha}]))$ whose initial data satisfies $\mathbf{c}(0,.) \in \mathscr{C}_{\mathcal{S}}(\Omega)$ is called a perfect mixture solution.

Note that the symmetry of coefficient $\alpha_{i j}$ (see (1.8)) imply that

$$
\partial_{t}\left(\sum_{i=1}^{n} c_{i}\right)=0
$$

Thus, a perfect mixture solution satisfies $\mathbf{c}(t,.) \in \mathscr{C}_{\mathcal{S}}(\Omega)$ for any $t \geqslant 0$.

Remark 1.1. Considering only perfect mixtures solutions, it is possible to remove one of the unknowns (and the associated evolution equation) from the system, for instance $c_{n}$, by simply setting $c_{n}=1-\sum_{i=1}^{n-1} c_{i}$. In the case $n=2$, we recover the fact that the system can be expressed using a single unknown, for instance $c=c_{1}=1-c_{2}$ (by symmetry the other choice gives exactly the same system). However, it is preferable to keep all the unknowns in the derivation and analysis of the models to avoid breaking the symmetry of the problem, which is one the key feature we want to preserve. Of course, from a numerical point of view one may only solve $n-1$ equations a posteriori, once it is proved that the numerical scheme which is used is coherent with perfect mixture solutions, that is to say that the relation $\sum_{i=1}^{n} c_{i}=1$ is preserved by the numerical method, see Refs. 3,6 .

Remark 1.2. The perfect mixture solutions of System $(\mathrm{CH}[\mathcal{F}, \boldsymbol{\alpha}])$ only depend on the values of the total free energy $\mathcal{F}$ on the hyperplane $\mathscr{C}_{\mathcal{S}}(\Omega)$.

In the sequel, we will also often make use of the following subspace

$$
\mathcal{H}=\left\{\left(\boldsymbol{\xi}_{1}, \ldots, \boldsymbol{\xi}_{n}\right) \in\left(\mathbb{R}^{d}\right)^{n}, \sum_{i=1}^{n} \boldsymbol{\xi}_{i}=0\right\} .
$$

since, if we set, for any $\mathbf{c} \in \mathscr{C}(\Omega)$,

$$
\nabla \mathbf{c}(x)=\left(\nabla c_{1}(x), \ldots, \nabla c_{n}(x)\right), \quad \forall x \in \Omega,
$$

we observe that

$$
\mathbf{c} \in \mathscr{C}_{\mathcal{S}}(\Omega) \Longrightarrow \nabla \mathbf{c}(x) \in \mathcal{H}, \quad \forall x \in \Omega
$$




\section{First step towards consistent $n$-phase Cahn-Hilliard systems}

\subsection{The consistency issue}

We consider now mixtures of $n$ constituents, $n \geqslant 2$. We suppose given the interface width $\varepsilon>0$ (which is assumed to be the same for each pair of phases in the mixture) and all the surface tensions $\sigma_{i j}=\sigma_{j i}>0$ between all the possible pairs of phases in the system, that we collect into a symmetric matrix $\boldsymbol{\sigma}=\left(\sigma_{i j}\right)_{i, j}$, with the convention that $\sigma_{i i}=0$ for any $i \in\{1, \ldots, n\}$. For a two-phase system, the matrix $\boldsymbol{\sigma}$ simply reads

$$
\boldsymbol{\sigma}=\left(\begin{array}{cc}
0 & \sigma \\
\sigma & 0
\end{array}\right)
$$

Our goal is to investigate the behavior of $n$-phase Cahn-Hilliard systems $(\mathrm{CH}[\mathcal{F}, \boldsymbol{\alpha}])$ in the case where some of the phases are absent in the initial state of the mixture. That's the reason why we introduce the following notation.

\section{Definition 2.1.}

- For any set of indices $I \subset\{1, \ldots, n\}$ with $|I|=k \leqslant n-2$, we define $\widetilde{\boldsymbol{\sigma}}^{I}$ to be the $(n-k) \times(n-k)$ surface tension matrix obtained from $\boldsymbol{\sigma}$ by removing the rows and columns whose index belongs to $I$.

For $I=\{l\}, l \in\{1, \ldots, n\}$, we simply write $\widetilde{\boldsymbol{\sigma}}^{l}$ instead of $\tilde{\boldsymbol{\sigma}}^{\{l\}}$.

- For any set of indices $I \subset\{1, \ldots, n\}$, with $|I|=k \leqslant n-2$, and for any $\mathbf{c} \in \mathbb{R}^{n}$ we define by $\widetilde{\mathbf{c}}^{I}$ the vector in $\mathbb{R}^{n-k}$ obtained from $\mathbf{c}$ by removing the entries whose index belongs to $I$.

For $I=\{l\}, l \in\{1, \ldots, n\}$, we simply write $\widetilde{\mathbf{c}}^{l}$ instead of $\widetilde{\mathbf{c}}^{\{l\}}$.

For any $n \geqslant 2$ and any choice of the surface tension matrix $\boldsymbol{\sigma}$, we would like to build a total free energy functional $\mathcal{F}_{\varepsilon}^{[\boldsymbol{\sigma}]}$ and diffusion coefficients $\boldsymbol{\alpha}^{[\boldsymbol{\sigma}]}=\left(\alpha_{i j}^{[\boldsymbol{\sigma}]}\right)_{i j}$ so that the associated systems $\left(\mathrm{CH}\left[\mathcal{F}_{\varepsilon}^{[\boldsymbol{\sigma}]}, \boldsymbol{\alpha}^{[\boldsymbol{\sigma}]}\right]\right)$ satisfy the following properties, refered to as consistency properties

$\left(\mathcal{C}_{1}\right)$ In the case $n=2$, with $\boldsymbol{\sigma}$ defined by $(2.1)$, we require that

$$
\mathcal{F}_{\varepsilon}^{[\boldsymbol{\sigma}]}(c, 1-c)=\mathcal{F}_{\varepsilon}^{\sigma}(c), \quad \forall c \in \mathscr{C}(\Omega),
$$

where the two-phase energy $\mathcal{F}_{\varepsilon}^{\sigma}$ is defined in (1.5).

$\left(\mathcal{C}_{2}\right)$ For any $I \subset\{1, \ldots, n\}$ with $|I|=k \leqslant n-2$, we have

$$
\left(\mathbf{c} \in \mathscr{C}_{\mathcal{S}}(\Omega) \text { and } c_{l} \equiv 0, \forall l \in I\right) \Longrightarrow \mathcal{F}_{\varepsilon}^{[\boldsymbol{\sigma}]}(\mathbf{c})=\mathcal{F}_{\varepsilon}^{\left[\tilde{\boldsymbol{\sigma}}^{I}\right]}\left(\widetilde{\mathbf{c}}^{I}\right) \text {. }
$$

$\left(\mathcal{C}_{3}\right)$ For any $I \subset\{1, \ldots, n\}$ with $|I|=k \leqslant n-2$, for any perfect mixture solution c of $\left(\mathrm{CH}\left[\mathcal{F}_{\varepsilon}^{[\boldsymbol{\sigma}]}, \boldsymbol{\alpha}^{[\boldsymbol{\sigma}]}\right]\right)$ we have

$$
\left(c_{l}(0, .) \equiv 0, \forall l \in I\right) \Longrightarrow\left(c_{l}(t, .) \equiv 0, \forall t \geqslant 0, \forall l \in I\right) .
$$

Let us comment on those properties. 
- Property $\left(\mathcal{C}_{1}\right)$ means that the $n$-phase energy with $n=2$ exactly coincides with the reference two-phase Cahn-Hilliard energy (1.5).

- Property $\left(\mathcal{C}_{2}\right)$ means that the $n$-phase free energy is equal to the corresponding $(n-k)$-phase free energy when the phases whose label belongs to $I$ are absent in the system.

- Property $\left(\mathcal{C}_{3}\right)$ means that, if some phases are absent in the initial data they remain absent all along the time evolution of the solution.

Remark 2.1. Observe that, by induction, it is enough to check those properties in the case where $|I|=k=1$, that is when only one of the phases is supposed to be absent in the system.

One of the main goal of the rest of this paper is to show that, in many situations, it is possible to explicitly build Cahn-Hilliard systems that fulfill these properties. Moreover, we will show from those consistency assumptions that the systems obtained this way form a hierarchy in the sense that: for any $I \subset\{1, \ldots, n\}$ with $|I|=k \leqslant n-2$, the PDE system $\left(\mathrm{CH}\left[\mathcal{F}_{\varepsilon}^{\left[\widetilde{\boldsymbol{\sigma}}^{I}\right]}, \boldsymbol{\alpha}^{\left[\widetilde{\boldsymbol{\sigma}}^{I}\right]}\right]\right)$ can be seen as a subsystem of $\left(\mathrm{CH}\left[\mathcal{F}_{\varepsilon}^{[\boldsymbol{\sigma}]}, \boldsymbol{\alpha}^{[\boldsymbol{\sigma}]}\right]\right)$. A precise statement is given in Section 2.5.2.

We will also illustrate the importance of such consistency properties when such systems, coupled with the Navier-Stokes equations, are used in the phase-field modelling of multiphase flows (see Section 4.3.1).

\subsection{General form of the system}

Mimicking the structure of the two-phase energy (1.5), we introduce now the following ansatz for the $n$-phase free energy

$$
\mathcal{F}_{\varepsilon}^{[\boldsymbol{\sigma}]}(\mathbf{c})=\int_{\Omega} \varepsilon a Q^{[\boldsymbol{\sigma}]}(\nabla \mathbf{c}(x))+\frac{b}{\varepsilon} F^{[\boldsymbol{\sigma}]}(\mathbf{c}(x)) d x,
$$

where $Q^{[\boldsymbol{\sigma}]}$ is a quadratic form in $\left(\mathbb{R}^{d}\right)^{n}$ accounting for capillary effects and $F^{[\boldsymbol{\sigma}]}$ is a nonlinear potential term corresponding to the bulk free energy away from interfaces. Observe that those yet unknowns quantities both depend on the various surface tensions stored in the matrix $\boldsymbol{\sigma}$ but not on the interface thickness. This ansatz is reasonnable since we require the consistency property $\left(\mathcal{C}_{1}\right)$ to hold.

Let us first analyse the consequences of the consistency conditions $\left(\mathcal{C}_{1}\right)$ and $\left(\mathcal{C}_{2}\right)$ on the possible choices for $Q^{[\boldsymbol{\sigma}]}$ and $F^{[\boldsymbol{\sigma}]}$. For any $c \in \mathscr{C}(\Omega)$, and $1 \leqslant i, j \leqslant n$, with $i \neq j$, we introduce $\mathbf{c}^{i j} \in \mathscr{C}_{\mathcal{S}}(\Omega)$ defined as follows

$$
\left(\mathbf{c}^{i j}\right)_{k}= \begin{cases}c & \text { for } k=i, \\ 1-c & \text { for } k=j, \\ 0 & \text { for } k \notin\{i, j\} .\end{cases}
$$

Proposition 2.1. Conditions $\left(\mathcal{C}_{1}\right)$ and $\left(\mathcal{C}_{2}\right)$ imply that 
(1) The quadratic terms satisfy

$$
Q^{[\boldsymbol{\sigma}]}(\boldsymbol{\xi})=-\frac{1}{2} \sum_{i \neq j} \sigma_{i j} \boldsymbol{\xi}_{i} \cdot \boldsymbol{\xi}_{j}, \quad \forall \boldsymbol{\xi} \in \mathcal{H}
$$

(2) For any $i \neq j$ and any $c \in \mathscr{C}(\Omega)$,

$$
F^{[\boldsymbol{\sigma}]}\left(\mathbf{c}^{i j}\right)=\sigma_{i j} f(c) .
$$

(3) For any $1 \leqslant l \leqslant n$, for all $\mathbf{c} \in \mathcal{S}$,

$$
c_{l}=0 \quad \Longrightarrow \quad F^{[\boldsymbol{\sigma}]}(\mathbf{c})=F^{\left[\tilde{\boldsymbol{\sigma}}^{l}\right]}\left(\widetilde{\mathbf{c}}^{l}\right)
$$

Proof. We look for the quadratic term $Q^{[\boldsymbol{\sigma}]}$ under the following general form

$$
Q^{[\boldsymbol{\sigma}]}(\boldsymbol{\xi})=\sum_{i, j}\left(\boldsymbol{q}_{i j} \boldsymbol{\xi}_{i}\right) \cdot \boldsymbol{\xi}_{j}
$$

where, for any $1 \leqslant i, j \leqslant n, \boldsymbol{q}_{i j}$ is a symmetric $d \times d$ matrix (we recall that $d$ is the space dimension) and $\boldsymbol{q}_{i j}=\boldsymbol{q}_{j i}$, for any $i \neq j$. A straightforward computation shows that this definition leads to

$$
Q^{[\boldsymbol{\sigma}]}(\boldsymbol{\xi})=\frac{1}{2} \sum_{i \neq j}\left(\left(2 \boldsymbol{q}_{i j}-\boldsymbol{q}_{i i}-\boldsymbol{q}_{j j}\right) \boldsymbol{\xi}_{i}\right) \cdot \boldsymbol{\xi}_{j}+\left(\sum_{i=1}^{n} \boldsymbol{\xi}_{i}\right) \cdot\left(\sum_{i=1}^{n} \boldsymbol{q}_{i i} \boldsymbol{\xi}_{i}\right),
$$

in such a way that the second term vanishes for $\boldsymbol{\xi} \in \mathcal{H}$. Applying Condition $\left(\mathcal{C}_{2}\right)$ with $I=\{1, \ldots, n\} \backslash\{i, j\}$ and Condition $\left(\mathcal{C}_{1}\right)$ we find that, for any $c \in \mathscr{C}(\Omega)$,

$$
\begin{aligned}
\mathcal{F}_{\varepsilon}^{[\boldsymbol{\sigma}]}\left(\mathbf{c}^{i j}\right) & =\int_{\Omega}-\varepsilon a\left(\left(2 \boldsymbol{q}_{i j}-\boldsymbol{q}_{i i}-\boldsymbol{q}_{j j}\right) \nabla c\right) \cdot \nabla c+\frac{b}{\varepsilon} F^{[\boldsymbol{\sigma}]}\left(\mathbf{c}^{i j}\right) d x \\
& =\int_{\Omega} \sigma_{i j} \varepsilon a|\nabla c|^{2}+\frac{b}{\varepsilon} \sigma_{i j} f(c) d x=\mathcal{F}_{\varepsilon}^{\sigma_{i j}}(c) .
\end{aligned}
$$

Such an equality can hold for any $c \in \mathscr{C}(\Omega)$ if and only if both gradient terms coincide as well as potential terms. This proves (2.3) and the relations

$$
\boldsymbol{q}_{i i}+\boldsymbol{q}_{j j}-2 \boldsymbol{q}_{i j}=\sigma_{i j} \mathrm{Id}, \quad \forall i \neq j .
$$

From (2.5) it follows that

$$
Q^{[\boldsymbol{\sigma}]}(\boldsymbol{\xi})=-\frac{1}{2} \sum_{\substack{i, j=1 \\ i \neq j}}^{n} \sigma_{i j} \boldsymbol{\xi}_{i} \cdot \boldsymbol{\xi}_{j}+\left(\sum_{i=1}^{n} \boldsymbol{\xi}_{i}\right) \cdot\left(\sum_{i=1}^{n} \boldsymbol{q}_{i i} \boldsymbol{\xi}_{i}\right),
$$

and since the second term vanishes on $\mathcal{H},(2.2)$ is proved. From (2.2), we deduce that

$$
Q^{[\boldsymbol{\sigma}]}(\nabla \mathbf{c})=Q^{\left[\widetilde{\boldsymbol{\sigma}}^{l}\right]}\left(\nabla \widetilde{\mathbf{c}}^{l}\right), \quad \forall \mathbf{c} \in \mathscr{C}_{\mathcal{S}}(\Omega) \text {, s.t. } c_{l}=0 .
$$

Therefore, applying condition $\left(\mathcal{C}_{2}\right)$ with $I=\{l\}$ leads to

$$
\int_{\Omega} F^{[\boldsymbol{\sigma}]}(\mathbf{c}) d x=\int_{\Omega} F^{\left[\widetilde{\boldsymbol{\sigma}}^{l}\right]}\left(\widetilde{\mathbf{c}}^{l}\right) d x, \quad \forall \mathbf{c} \in \mathscr{C}_{\mathcal{S}}(\Omega) \text {, s.t. } c_{l}=0 .
$$


This implies the pointwise equality (2.4) of the potentials.

Remark 2.2. Thanks to Remark 1.2 the values of $Q^{[\boldsymbol{\sigma}]}$ outside $\mathcal{H}$ have no influence on the perfect mixture solutions of the system that we are interested in. Therefore, without loss of generality, we shall define in the sequel

$$
Q^{[\boldsymbol{\sigma}]}(\boldsymbol{\xi})=-\frac{1}{2} \sum_{\substack{i, j=1 \\ i \neq j}}^{n} \sigma_{i j} \boldsymbol{\xi}_{i} \cdot \boldsymbol{\xi}_{j}, \quad \forall \boldsymbol{\xi} \in\left(\mathbb{R}^{d}\right)^{n}
$$

\subsection{Admissibility conditions for capillary terms}

Given the surface tensions $\boldsymbol{\sigma}$, we determined in Proposition 2.1 the general form that the quadratic terms in the free energy has to take.

It is now clear that for such a free energy to be admissible from a physical point of view, as well a mathematical point of view, it is required that the contribution of those quadratic terms in the energy is positive in the interfaces. If not, the free energy will not be bounded from below and the system will be mathematically ill-posed. This coercivity assumption means that the property

$$
Q^{[\boldsymbol{\sigma}]}(\boldsymbol{\xi}) \geqslant C_{\boldsymbol{\sigma}} \sum_{i=1}^{n}\left|\boldsymbol{\xi}_{i}\right|^{2}, \quad \forall \boldsymbol{\xi} \in \mathcal{H}
$$

has to be satisfied for some $C_{\boldsymbol{\sigma}}>0$. From (2.2), this is equivalent to the following admissibility condition for the surface tensions

$$
{ }^{t} \mathbf{x} \boldsymbol{\sigma} \mathbf{x} \leqslant-C_{\boldsymbol{\sigma}}|\mathbf{x}|^{2}, \quad \forall \mathbf{x} \in \mathbb{R}^{n} \text {, s. t. } \mathbf{x} \cdot \mathbf{1}=0 \text {, with } \mathbf{1}={ }^{t}(1, \ldots, 1) \in \mathbb{R}^{n} .
$$

We shall see below that this assumption is central in the construction and the analysis of the Cahn-Hilliard models that we propose in this paper. Without this structural assumption on the surface tensions, we do not know how to deal with such Cahn-Hilliard type models. This issue was already pointed out for three-component systems in Ref. 3 and is not specific to the number of components we consider.

Let us have a closer look at Condition (2.6) in some simple cases. For $n=2, \boldsymbol{\sigma}$ is given by (2.1), and we easily see that assumption (2.6) reduces to the condition $\sigma>0$ which is physically relevant since surface tensions are positive quantities. For $n=3$, one can check that Condition (2.6) is equivalent to

$$
\sigma_{12}>0, \sigma_{13}>0, \sigma_{23}>0, \text { and } \Sigma_{1} \Sigma_{2}+\Sigma_{1} \Sigma_{3}+\Sigma_{2} \Sigma_{3}>0,
$$

where $\Sigma_{i} \stackrel{\text { def }}{=} \sigma_{i j}+\sigma_{i k}-\sigma_{k j}, \quad\{i, j, k\}=\{1,2,3\}$ is the opposite of the so-called spreading coefficient of phase $i$ in the system. We recover here the conditions obtained in Ref. 3. For $n=4$, the situation is a bit more complex and Condition (2.6) is equivalent to the three properties

$$
\begin{gathered}
\sigma_{i j}>0, \quad \forall 1 \leqslant i<j \leqslant 4, \\
\Delta_{l} \stackrel{\text { def }}{=}{\widetilde{\Sigma_{i}}}^{l}{\widetilde{\Sigma_{j}}}^{l}+{\widetilde{\Sigma_{i}}}^{l}{\widetilde{\Sigma_{k}}}^{l}+{\widetilde{\Sigma_{j}}}^{l}{\widetilde{\Sigma_{k}}}^{l}>0, \text { for any }\{i, j, k, l\}=\{1,2,3,4\},
\end{gathered}
$$




$$
\Delta_{i} \Delta_{j}>\left(2 \sigma_{k l}{\widetilde{\Sigma_{k}}}^{l}-{\widetilde{\Sigma_{k}}}^{i}{\widetilde{\Sigma_{k}}}^{j}\right)^{2} \text {, for any }\{i, j, k, l\}=\{1,2,3,4\} .
$$

In those formulas we defined $\Sigma_{i}^{l}$ as in the case $n=3$ by assuming that the phase $l$ is absent, that is ${\widetilde{\Sigma_{i}}}_{i}^{l} \stackrel{\text { def }}{=} \sigma_{i j}+\sigma_{i k}-\sigma_{k j}, \quad\{i, j, k, l\}=\{1,2,3,4\}$.

Finally, observe that for arbitrary $n \geqslant 2$ and if all the surface tensions are equal, e.g. $\sigma_{i j}=\sigma>0, \forall i \neq j$, then a straightforward computation shows that (2.6) is automatically satisfied with $C_{\sigma}=\sigma$.

\subsection{Diffusion coefficients. Mobility}

We are now going to discuss the possible choices of the diffusion coefficients $\alpha_{i j}^{[\boldsymbol{\sigma}]}$ appearing in the system $\left(\mathrm{CH}\left[\mathcal{F}_{\varepsilon}^{[\boldsymbol{\sigma}]}, \boldsymbol{\alpha}^{[\boldsymbol{\sigma}]}\right]\right)$ in order to satisfy the consistency assumptions. We recall that these diffusion coefficients have to fulfill the constitutive relations (1.8),(1.9) and (1.10). Let us first state the following technical lemma, whose proof is given in Appendix B.

Lemma 2.1. Assume that $\boldsymbol{\sigma}$ is such that (2.6) holds. Then, there exists a unique $n \times n$ matrix $\overline{\boldsymbol{\alpha}}^{[\boldsymbol{\sigma}]}$ and a unique vector $\boldsymbol{\gamma}^{[\boldsymbol{\sigma}]} \in \mathbb{R}^{n}$, such that

$$
\left\{\begin{array}{l}
\overline{\boldsymbol{\alpha}}^{[\boldsymbol{\sigma}]} \boldsymbol{\sigma}=\mathrm{Id}+\boldsymbol{\gamma}^{[\boldsymbol{\sigma}]} \otimes \mathbf{1}, \\
\overline{\boldsymbol{\alpha}}^{[\boldsymbol{\sigma}]} \mathbf{1}=0 .
\end{array}\right.
$$

with $\mathbf{1}={ }^{t}(1, \ldots, 1) \in \mathbb{R}^{n}$. Moreover, $\overline{\boldsymbol{\alpha}}^{[\boldsymbol{\sigma}]}$ is symmetric and satisfies the following properties

$$
\overline{\boldsymbol{\alpha}}^{[\boldsymbol{\sigma}]} \boldsymbol{\sigma} \overline{\boldsymbol{\alpha}}^{[\boldsymbol{\sigma}]}=\overline{\boldsymbol{\alpha}}^{[\boldsymbol{\sigma}]},
$$

$$
\operatorname{Ker} \overline{\boldsymbol{\alpha}}^{[\boldsymbol{\sigma}]}=\operatorname{Span}\{\mathbf{1}\} .
$$

We are now going to prove that, in this framework, there exists one single suitable choice for the diffusion coefficients up to a multiplicative constant.

Proposition 2.2. Assume that we are given a surface tension matrix $\boldsymbol{\sigma}$ satisfying the coercivity assumption (2.6). Then, a necessary condition for the consistency condition $\left(\mathcal{C}_{3}\right)$ to hold true is that there exists $M_{\boldsymbol{\sigma}} \in \mathbb{R}^{*}$ such that

$$
\boldsymbol{\alpha}^{[\boldsymbol{\sigma}]}=M_{\boldsymbol{\sigma}} \overline{\boldsymbol{\alpha}}^{[\boldsymbol{\sigma}]} .
$$

This diffusion coefficient $M_{\boldsymbol{\sigma}}$ is called the mobility.

Proof. Observe that Condition $\left(\mathcal{C}_{3}\right)$ is equivalent to the following property

$$
\mathbf{c} \in \mathscr{C}_{\mathcal{S}}(\Omega) \text {, with } c_{i} \equiv 0 \Longrightarrow \sum_{j=1}^{n} \alpha_{i j}^{[\sigma]} \mu_{j}=\text { Cte }
$$

that is required to hold for any $i \in\{1, \ldots, n\}$ (see Remark 2.1). 
Let us fix some $i \in\{1, \ldots, n\}$ and we compute

$$
\sum_{j=1}^{n} \alpha_{i j}^{[\boldsymbol{\sigma}]} \mu_{j}=\frac{a}{\varepsilon} \sum_{j=1}^{n} \alpha_{i j}^{[\boldsymbol{\sigma}]} \partial_{j} F^{[\boldsymbol{\sigma}]}(\mathbf{c})+\frac{b}{2} \varepsilon \sum_{j=1}^{n}\left(\boldsymbol{\alpha}^{[\boldsymbol{\sigma}]} \boldsymbol{\sigma}\right)_{i j} \Delta c_{j} .
$$

Property (2.9) says that this term is required to be independent of $x$ for any choice of $\mathbf{c} \in \mathscr{C}_{\mathcal{S}}(\Omega)$ satisfying $c_{i} \equiv 0$. It is clear that the nonlinear potential term should be constant and that the second order differential term should vanish identically. Therefore, it is in particular needed that

$$
\sum_{j=1}^{n}\left(\boldsymbol{\alpha}^{[\boldsymbol{\sigma}]} \boldsymbol{\sigma}\right)_{i j} \Delta c_{j} \equiv 0, \quad \forall \mathbf{c} \in \mathscr{C}_{\mathcal{S}}(\Omega), \text { s.t. } c_{i} \equiv 0 .
$$

Let us choose some $k \in\{1, \ldots, n\}$ different from $i$. Since $\sum_{j=1}^{n} c_{j} \equiv 1$ and $c_{i}=0$, we have

$$
\Delta c_{k}=-\sum_{\substack{j=1 \\ j \neq k, j \neq i}}^{n} \Delta c_{j}
$$

so that the left-hand side term in (2.10) can be written as follows

$$
\sum_{j=1}^{n}\left(\boldsymbol{\alpha}^{[\boldsymbol{\sigma}]} \boldsymbol{\sigma}\right)_{i j} \Delta c_{j}=\sum_{\substack{j=1 \\ j \neq i, j \neq k}}^{n}\left(\left(\boldsymbol{\alpha}^{[\boldsymbol{\sigma}]} \boldsymbol{\sigma}\right)_{i j}-\left(\boldsymbol{\alpha}^{[\boldsymbol{\sigma}]} \boldsymbol{\sigma}\right)_{i k}\right) \Delta c_{j} .
$$

Therefore, condition (2.10) amounts to ask that this quantity vanishes for any choice of the functions $c_{j} \in \mathscr{C}(\Omega), j \notin\{i, k\}$ ( $c_{i}$ is supposed to be 0 , and $c_{k}$ is determined by using that $\mathbf{c}(x) \in \mathcal{S}$, that is $\left.c_{k}(x)=1-\sum_{j \neq k} c_{j}(x)\right)$.

It follows that the following algebraic condition has to be satisfied

$$
\left(\boldsymbol{\alpha}^{[\boldsymbol{\sigma}]} \boldsymbol{\sigma}\right)_{i j}=\left(\boldsymbol{\alpha}^{[\boldsymbol{\sigma}]} \boldsymbol{\sigma}\right)_{i k}, \quad \forall j, k \in\{1, \ldots, n\} \backslash\{i\} .
$$

Hence, it is needed that for some $\gamma_{i}, d_{i} \in \mathbb{R}$ depending only on $i$, we have

$$
\left\{\begin{array}{l}
\left(\boldsymbol{\alpha}^{[\boldsymbol{\sigma}]} \boldsymbol{\sigma}\right)_{i j}=\gamma_{i}, \\
\left(\boldsymbol{\alpha}^{[\boldsymbol{\sigma}]} \boldsymbol{\sigma}\right)_{i i}=d_{i}+\gamma_{i},
\end{array} \quad \forall j \neq i\right.
$$

Introducing the diagonal matrix $D=\operatorname{diag}\left(d_{1}, \ldots, d_{n}\right)$ and the vector $\gamma=\left(\gamma_{i}\right)_{1 \leqslant i \leqslant n}$, we finally arrive to the matrix equation

$$
\boldsymbol{\alpha}^{[\boldsymbol{\sigma}]} \boldsymbol{\sigma}=D+\boldsymbol{\gamma} \otimes \mathbf{1}
$$

that should be satisfied by $\boldsymbol{\alpha}^{[\boldsymbol{\sigma}]}$. Moreover $\boldsymbol{\alpha}^{[\boldsymbol{\sigma}]}$ has to be symmetric (see (1.8)), to satisfy that $\boldsymbol{\alpha}^{[\boldsymbol{\sigma}]} \mathbf{1}=0$ (see (1.9)) and should not have any row (or column) which is identically zero (see $(1.10)$ ).

Right-multiplying the matrix equation (2.11) by $\boldsymbol{\alpha}^{[\boldsymbol{\sigma}]}$, and using that $\boldsymbol{\alpha}^{[\boldsymbol{\sigma}]} \mathbf{1}=0$, we obtain

$$
\boldsymbol{\alpha}^{[\boldsymbol{\sigma}]} \boldsymbol{\sigma} \boldsymbol{\alpha}^{[\boldsymbol{\sigma}]}=D \boldsymbol{\alpha}^{[\boldsymbol{\sigma}]}
$$


Let us show that $d_{i} \neq 0$ for any $i$. Indeed, if for some $i$ we have $d_{i}=0$, then we arrive to

$$
{ }^{t} \boldsymbol{e}_{i} \boldsymbol{\alpha}^{[\boldsymbol{\sigma}]} \boldsymbol{\sigma} \boldsymbol{\alpha}^{[\boldsymbol{\sigma}]} \boldsymbol{e}_{i}=0,
$$

where $\left(\boldsymbol{e}_{i}\right)_{i}$ is the canonical basis of $\mathbb{R}^{n}$. Since $\left(\boldsymbol{\alpha}^{[\boldsymbol{\sigma}]} \boldsymbol{e}_{i}\right) \cdot \mathbf{1}=\boldsymbol{e}_{i} \cdot\left(\boldsymbol{\alpha}^{[\boldsymbol{\sigma}]} \mathbf{1}\right)=0$, the coercivity assumption (2.6) implies that $\boldsymbol{\alpha}^{[\boldsymbol{\sigma}]} \boldsymbol{e}_{i}=0$, that is the $i$ th row of $\boldsymbol{\alpha}^{[\boldsymbol{\sigma}]}$ is zero, which is not possible.

We can now left-multiply the equation (2.11) by the inverse of the matrix $D$ and use the uniqueness property stated in Lemma 2.1 to conclude that

$$
\boldsymbol{\alpha}^{[\boldsymbol{\sigma}]}=D \overline{\boldsymbol{\alpha}}^{[\boldsymbol{\sigma}]} .
$$

The three matrices involved in this inequality are symmetric and the transpose of this equation leads to

$$
\boldsymbol{\alpha}^{[\boldsymbol{\sigma}]}=\overline{\boldsymbol{\alpha}}^{[\boldsymbol{\sigma}]} D
$$

Right-multiplying this equality by $\mathbf{1}$ proves that the vector $D \mathbf{1}$ belongs to $\operatorname{Ker} \overline{\boldsymbol{\alpha}}^{[\boldsymbol{\sigma}]}$. Owing to Lemma 2.1, this proves that

$$
D \mathbf{1} \in \operatorname{Span}\{\mathbf{1}\} .
$$

This exactly means that there exists $M_{\boldsymbol{\sigma}} \in \mathbb{R}$ such that $D=M_{\sigma} \mathrm{Id}$, and consequently that

$$
\boldsymbol{\alpha}^{[\boldsymbol{\sigma}]}=M_{\boldsymbol{\sigma}} \overline{\boldsymbol{\alpha}}^{[\boldsymbol{\sigma}]}
$$

We can now prove that $M_{\boldsymbol{\sigma}}$ needs to be positive.

Proposition 2.3. Assume that we are given a surface tension matrix $\boldsymbol{\sigma}$ satisfying the coercivity assumption (2.6). The n-phase Cahn-Hilliard system $\left(\mathrm{CH}\left[\mathcal{F}_{\varepsilon}^{[\boldsymbol{\sigma}]}, \boldsymbol{\alpha}^{[\boldsymbol{\sigma}]}\right]\right)$, with $\boldsymbol{\alpha}^{[\boldsymbol{\sigma}]}=M_{\boldsymbol{\sigma}} \overline{\boldsymbol{\alpha}}^{[\boldsymbol{\sigma}]}$ is thermodynamically consistent (that is to say dissipative) if and only if $M_{\boldsymbol{\sigma}}>0$.

Proof. We use (2.8) to deduce that the right-hand side in the formal energy inequality (1.11), refered to as $\mathcal{D}$, can be written

$$
\begin{aligned}
\mathcal{D} & \stackrel{\text { def }}{=} \sum_{1 \leqslant i, j \leqslant n} \int_{\Omega} \alpha_{i j}^{[\boldsymbol{\sigma}]} \nabla \mu_{i} \cdot \nabla \mu_{j} d x \\
& =\sum_{1 \leqslant i, j \leqslant n} \int_{\Omega} M_{\boldsymbol{\sigma}} \bar{\alpha}_{i j}^{[\boldsymbol{\sigma}]} \nabla \mu_{i} \cdot \nabla \mu_{j} d x \\
& =\sum_{1 \leqslant k, l \leqslant n} \sigma_{k l} \int_{\Omega} M_{\boldsymbol{\sigma}}\left(\sum_{i=1}^{n} \bar{\alpha}_{k i}^{[\boldsymbol{\sigma}]} \nabla \mu_{i}\right) \cdot\left(\sum_{j=1}^{n} \bar{\alpha}_{l j}^{[\boldsymbol{\sigma}]} \nabla \mu_{j}\right) d x \\
& =\sum_{1 \leqslant k, l \leqslant n} \sigma_{k l} \int_{\Omega} \frac{1}{M_{\boldsymbol{\sigma}}} \boldsymbol{J}_{k} \cdot \boldsymbol{J}_{l} d x,
\end{aligned}
$$


where $\boldsymbol{J}_{k}$ stands for the total diffusion flux of the phase $k$ defined by (1.6). Since $\sum_{k=1}^{n} \boldsymbol{J}_{k}=0$, by using assumption (2.6), we finally find that this term is nonnegative if and only if $M_{\boldsymbol{\sigma}}$ is positive. Moreover, we have the estimate

$$
\mathcal{D} \leqslant-C_{\boldsymbol{\sigma}}\left(\sum_{k=1}^{n} \int_{\Omega} \frac{1}{M_{\boldsymbol{\sigma}}}\left|\boldsymbol{J}_{k}\right|^{2} d x\right),
$$

which proves that the total dissipation vanishes if and only if all the total mass fluxes $\boldsymbol{J}_{k}, k=1, \ldots, n$ are zero, that is at the thermodynamical equilibrium.

\subsection{Intermediate conclusions}

\subsubsection{The n-phase Cahn-Hilliard model}

From now on, we have proved that

- The surface tensions should satisfy (2.6).

- The diffusion coefficients in the system should be of the form

$$
\boldsymbol{\alpha}^{[\boldsymbol{\sigma}]}=M_{\sigma} \overline{\boldsymbol{\alpha}}^{[\boldsymbol{\sigma}]},
$$

with $M_{\boldsymbol{\sigma}}>0$ and $\overline{\boldsymbol{\alpha}}^{[\boldsymbol{\sigma}]}$ uniquely defined from $\boldsymbol{\sigma}$ by Lemma 2.1. Observe that, in general, simple explicit formulas for $\overline{\boldsymbol{\alpha}}^{[\sigma]}$ are not available, but in practice these coefficients can be easily computed by solving numerically the linear system (2.7). Notice that, if necessary, we may allow $M_{\boldsymbol{\sigma}}$ to depend on $\mathbf{c}$ : it does not change the formal derivation of the model but may imply new mathematical and numerical issues that we do not want to describe extensively here (see the introduction of Section 4).

- The potential $F^{[\boldsymbol{\sigma}]}$ should satisfy at least Properties (2.3) and (2.4).

In particular, the $n$-phase free energy we obtain reads

$$
\mathcal{F}_{\varepsilon}^{[\boldsymbol{\sigma}]}(\mathbf{c})=\int_{\Omega} \frac{b}{\varepsilon} F^{[\boldsymbol{\sigma}]}(\mathbf{c}(x))-\varepsilon \frac{a}{2} \sum_{i \neq j} \sigma_{i j} \nabla c_{i} \cdot \nabla c_{j} d x .
$$

The associated Cahn-Hilliard system $\left(\mathrm{CH}\left[\mathcal{F}_{\varepsilon}^{[\boldsymbol{\sigma}]}, \boldsymbol{\alpha}^{[\boldsymbol{\sigma}]}\right]\right)$ can be written in the following form

$$
\left\{\begin{aligned}
\partial_{t} c_{i} & =-\operatorname{div}\left(M_{\boldsymbol{\sigma}} \nabla\left(\sum_{j} \bar{\alpha}_{i j}^{[\boldsymbol{\sigma}]} \mu_{j}\right)\right), \\
\mu_{i} & =\frac{\delta \mathcal{F}_{\varepsilon}^{[\boldsymbol{\sigma}]}}{\delta c_{i}}=\frac{b}{\varepsilon} \partial_{i} F^{[\boldsymbol{\sigma}]}+\frac{a}{2} \varepsilon \sum_{1 \leqslant j \leqslant n} \sigma_{i j} \Delta c_{j} .
\end{aligned}\right.
$$

Equivalently, if we want to get rid of the variables $\left(\mu_{i}\right)_{i}$, we can write

$$
\partial_{t} c_{i}=\operatorname{div}\left(M_{\boldsymbol{\sigma}} \nabla\left(\frac{b}{\varepsilon} L_{i}^{[\boldsymbol{\sigma}]}\left(F^{[\boldsymbol{\sigma}]}\right)(\mathbf{c})-\frac{a}{2} \varepsilon \Delta c_{i}\right)\right),
$$


where we have introduced the following differential operators (acting on CahnHilliard bulk phase potentials $F: \mathbb{R}^{n} \rightarrow \mathbb{R}$ )

$$
L_{i}^{[\boldsymbol{\sigma}]}(F)=-\sum_{j=1}^{n} \bar{\alpha}_{i j}^{[\boldsymbol{\sigma}]} \partial_{j} F, \quad \forall i=1, \ldots, n .
$$

It remains to make explicit the expression of the potential $F^{[\boldsymbol{\sigma}]}$. Note that, whatever the expression of $F^{[\boldsymbol{\sigma}]}$ is, Properties (2.3) and (2.4) are sufficient to ensure the consistency properties $\left(\mathcal{C}_{1}\right)$ and $\left(\mathcal{C}_{2}\right)$. To guarantee the property $\left(\mathcal{C}_{3}\right)$ we impose the additional following sufficient condition on the potential $F^{[\sigma]}$

$$
\forall 1 \leqslant l \leqslant n, \forall \mathbf{c} \in \mathcal{S}, \quad c_{l}=0 \quad \Longrightarrow \quad L_{l}^{[\boldsymbol{\sigma}]}\left(F^{[\boldsymbol{\sigma}]}\right)(\mathbf{c})=0 .
$$

Section 3 will be entirely dedicated to the problem of finding suitable expressions of the potentials $F^{[\sigma]}$ in order to fulfill all the requirements we have listed so far.

Remark 2.3. In the case $n=3$, the model (2.12) is exactly the one proposed in Ref. 3. Indeed, in that case, the coefficients $\bar{\alpha}_{i j}^{[\sigma]}$ can be expressed thanks to the (opposites of the) three spreading coefficients $\Sigma_{1}=\sigma_{12}+\sigma_{13}-\sigma_{23}, \Sigma_{2}=$ $\sigma_{12}+\sigma_{23}-\sigma_{13}$ and $\Sigma_{3}=\sigma_{13}+\sigma_{23}-\sigma_{12}$ as follows

$$
\bar{\alpha}_{i j}^{[\boldsymbol{\sigma}]}=\frac{\Sigma_{T}}{3} \frac{1}{\Sigma_{i} \Sigma_{j}}, \quad \forall 1 \leqslant i \neq j \leqslant 3,
$$

with $\Sigma_{T}$ defined by $\frac{3}{\Sigma_{T}}=\frac{1}{\Sigma_{1}}+\frac{1}{\Sigma_{2}}+\frac{1}{\Sigma_{3}}$.

\subsubsection{Hierarchic structure of the models}

Assuming for the moment that we have at our disposal Cahn-Hilliard potentials $F^{[\boldsymbol{\sigma}]}$ satisfying Properties (2.3), (2.4) and (2.14), we can prove that the familly of models defined by (2.12) is a hierarchy in the following sense: if $\mathbf{c}$ is a particular solution such that, for some $1 \leqslant l \leqslant n, c_{l} \equiv 0$ (recall that the consistency conditions we have introduced exactly ensure that this is equivalent to $\left.c_{l}(t=0)=0\right)$ then it is also a solution of the suitable underlying $(n-1)$-phase Cahn-Hilliard model. The precise result we prove is the following.

Proposition 2.4. Let $n \geqslant 3$ and $I \subset\{1, \ldots, n\}$ with $|I| \leqslant n-2$. Assume that the potential $F^{[\boldsymbol{\sigma}]}$ satisfies Properties (2.3), (2.4) and (2.14). Consider a particular solution $\mathbf{c}$ of the n-phase Cahn-Hilliard model associated with $\boldsymbol{\sigma}$ satisfying $c_{l} \equiv$ $0, \forall l \in I$. Then, $\widetilde{\mathbf{c}}^{I}$ is a solution of the $(n-|I|)$-phase Cahn-Hilliard model associated with $\widetilde{\boldsymbol{\sigma}}^{I}$.

We will use the following lemma, whose proof is postponed to Appendix B.

Lemma 2.2. The diagonal entries of the matrix $\overline{\boldsymbol{\alpha}}^{[\boldsymbol{\sigma}]}$ are not zero. Moreover, the following formula holds

$$
\bar{\alpha}_{\tilde{i}^{\imath} \widetilde{j}^{l}}^{\left[\widetilde{\boldsymbol{\sigma}}^{l}\right]}=\bar{\alpha}_{i j}^{[\boldsymbol{\sigma}]}-\frac{\bar{\alpha}_{i l}^{[\boldsymbol{\sigma}]} \bar{\alpha}_{j l}^{[\boldsymbol{\sigma}]}}{\bar{\alpha}_{l l}^{[\boldsymbol{\sigma}]}}, \quad \forall 1 \leqslant i, j, l \leqslant n, i \neq l, j \neq l,
$$


where we have introduced the following notation for any $k \neq l$

$$
\tilde{k}^{l}=k, \text { if } k<l \quad \text { and } \quad \tilde{k}^{l}=k-1 \text {, if } k>l .
$$

Proof (of Proposition 2.4). By induction, it is sufficient to prove the result for $|I|=1$. Let $k$ and $l$ two distinct integers such that $1 \leqslant l \neq k \leqslant n$. We prove that, if $c_{l} \equiv 0$, the $k$-th equation of the $n$-phase Cahn-Hilliard model associated with $\boldsymbol{\sigma}$ is exactly the same as the $\widetilde{k}^{l}$-th equation of the (n-1)-phase Cahn-Hilliard model associated with $\tilde{\boldsymbol{\sigma}}^{l}$, where $\tilde{k}^{l}$ is defined in (2.16).

Owing to the formulation (2.12) of the system, it is sufficient to prove that

$$
L_{k}^{[\boldsymbol{\sigma}]}\left(F^{[\boldsymbol{\sigma}]}\right)(\mathbf{c})=L_{\widetilde{k}^{l}}^{\left[\widetilde{\boldsymbol{\sigma}}^{l}\right]}\left(F^{\left[\widetilde{\boldsymbol{\sigma}}^{l}\right]}\right)\left(\widetilde{\mathbf{c}}^{l}\right), \quad \forall \mathbf{c} \in \mathcal{S} \text { s.t. } c_{l}=0 .
$$

To this end and keeping in mind the definition of $L_{k}^{[\boldsymbol{\sigma}]}$, we first evaluate each partial derivative of the potential $F^{[\boldsymbol{\sigma}]}$ at a point $\mathbf{c} \in \mathcal{S}$ such that $c_{l}=0$. The $s$-th partial derivatives of $F^{[\sigma]}$ with $s \neq l$ can be directly obtained by differentiating the equality (2.4) with respect to $c_{s}$. We find

$$
\partial_{s} F^{[\boldsymbol{\sigma}]}(\mathbf{c})=\partial_{\widetilde{s}^{l}} F^{\left[\widetilde{\boldsymbol{\sigma}}^{l}\right]}\left(\widetilde{\mathbf{c}}^{l}\right), \quad \forall 1 \leqslant s \neq l \leqslant n, \forall \mathbf{c} \in \mathcal{S} \text { s.t. } c_{l}=0 .
$$

To evaluate the $l$-th partial derivatives of $F^{[\boldsymbol{\sigma}]}$, we first make use of (2.18) to deduce that, for all $\mathbf{c} \in \mathcal{S}$ such that $c_{l}=0$,

$$
\begin{aligned}
L_{l}^{[\boldsymbol{\sigma}]}\left(F^{[\boldsymbol{\sigma}]}\right)(\mathbf{c}) & =-\bar{\alpha}_{l l}^{[\boldsymbol{\sigma}]} \partial_{l} F^{[\boldsymbol{\sigma}]}(\mathbf{c})-\sum_{\substack{1 \leqslant s \leqslant n \\
s \neq l}} \bar{\alpha}_{l s}^{[\boldsymbol{\sigma}]} \partial_{s} F^{[\boldsymbol{\sigma}]}(\mathbf{c}) \\
& =-\bar{\alpha}_{l l}^{[\boldsymbol{\sigma}]} \partial_{l} F^{[\boldsymbol{\sigma}]}(\mathbf{c})-\sum_{\substack{1 \leqslant s \leqslant n \\
s \neq l}} \bar{\alpha}_{l s}^{[\boldsymbol{\sigma}]} \partial_{\widetilde{s}^{l}} F^{\left[\widetilde{\boldsymbol{\sigma}}^{l}\right]}\left(\widetilde{\mathbf{c}}^{l}\right) .
\end{aligned}
$$

However, from Property (2.14), we know that $L_{l}^{[\boldsymbol{\sigma}]}\left(F^{[\boldsymbol{\sigma}]}\right)(\mathbf{c})=0$, so that we obtain

$$
\sum_{\substack{1 \leqslant s \leqslant n \\ s \neq l}} \bar{\alpha}_{l s}^{[\boldsymbol{\sigma}]} \partial_{\widetilde{s}^{l}} F^{\left[\widetilde{\boldsymbol{\sigma}}^{l}\right]}\left(\widetilde{\mathbf{c}}^{l}\right)=-\bar{\alpha}_{l l}^{[\boldsymbol{\sigma}]} \partial_{l} F^{[\boldsymbol{\sigma}]}(\mathbf{c}), \quad \forall \mathbf{c} \in \mathcal{S} \text { s.t. } c_{l}=0 .
$$

From (2.18) and (2.19), it is now easy to deduce that

$$
L_{k}^{[\boldsymbol{\sigma}]}\left(F^{[\boldsymbol{\sigma}]}\right)(\mathbf{c})=\sum_{\substack{1 \leqslant s \leqslant n \\ s \neq l}}\left[\bar{\alpha}_{k s}^{[\boldsymbol{\sigma}]}-\frac{\bar{\alpha}_{k l}^{[\boldsymbol{\sigma}]} \bar{\alpha}_{l s}^{[\boldsymbol{\sigma}]}}{\bar{\alpha}_{l l}^{[\boldsymbol{\sigma}]}}\right] \partial_{\widetilde{s}^{l}} F^{\left[\widetilde{\boldsymbol{\sigma}}^{l}\right]}\left(\widetilde{\mathbf{c}}^{l}\right), \quad \forall \mathbf{c} \in \mathcal{S} \text { s.t. } c_{l}=0 .
$$

The equality (2.17) is then deduced from Lemma 2.2.

\subsubsection{Well-posedness result}

We supplement the system (2.12) with Neumann boundary conditions for each order parameter $c_{i}$ and for each chemical potential $\mu_{i}$. That is, for $1 \leqslant i \leqslant n$,

$$
\nabla c_{i} \cdot \mathbf{n}=M_{\boldsymbol{\sigma}} \nabla \mu_{i} \cdot \mathbf{n}=0 .
$$


We choose the functional energy espace $\mathscr{C}(\Omega)=\left(H^{1}(\Omega)\right)^{n}$ and we assume that at the initial time, we have

$$
c_{i}(t=0)=c_{i}^{0}
$$

where $\mathbf{c}^{0} \in \mathscr{C}_{S}(\Omega)$ is given.

The existence and uniqueness of weak solutions of the problem (2.12) satisfying (2.20) and (2.21) can be proved in $2 \mathrm{D}$ and $3 \mathrm{D}$, under the following general assumptions

$$
\left\{\begin{array}{l}
F^{[\boldsymbol{\sigma}]} \in \mathcal{C}^{2}\left(\mathbb{R}^{n}\right), \text { and } \inf _{\mathbb{R}^{n}} F^{[\boldsymbol{\sigma}]}>-\infty, \\
\left|\partial^{\alpha} F^{[\boldsymbol{\sigma}]}(\mathbf{c})\right| \leqslant C\left(1+|\mathbf{c}|^{p-|\alpha|}\right), \forall \mathbf{c} \in \mathcal{S}, \forall|\alpha| \leqslant 2,
\end{array}\right.
$$

where $p \in[2, \infty[$ in $2 \mathrm{D}$, and $p \in[2,6]$ in $3 \mathrm{D}$.

Theorem 2.1. Assume that conditions (2.6) and (2.22) hold. Consider the problem (2.12) together with the initial condition (2.21) and the Neumann boundary conditions (2.20) for each unknowns $\left(c_{i}, \mu_{i}\right)$. Then, there exists a unique weak solution $(\mathbf{c}, \boldsymbol{\mu})$ on $[0,+\infty[$ such that

$$
\begin{gathered}
\mathbf{c} \in L^{\infty}\left(0,+\infty ;\left(H^{1}(\Omega)\right)^{n}\right) \cap C^{0}\left(\left[0,+\infty\left[;\left(L^{2}(\Omega)\right)^{n}\right),\right.\right. \\
\mathbf{c}(t, x) \in \mathcal{S}, \text { for a.e. }(t, x) \in[0,+\infty[\times \Omega, \\
\boldsymbol{\mu} \in L^{2}\left(0,+\infty ;\left(H^{1}(\Omega)\right)^{n}\right) .
\end{gathered}
$$

The proof of this result, based on a Galerkin approximation, suitable energy estimates and compactness results can be obtained by following exactly the same lines as in Ref. 3 where a similar result is proved in detail for $n=3$. The important point is that, in the present $n$-phase model, the energy estimate is guaranteed by (1.11), by the boundedness from below of $F^{[\boldsymbol{\sigma}]}$, by the coercivity assumption (2.6), and by the dissipativity property stated in Proposition 2.3 .

\section{Construction of consistent $\boldsymbol{n}$-phase Cahn-Hilliard potentials}

In this section, we give the details of the construction of $n$-phase Cahn-Hilliard potentials satisfying Properties (2.3), (2.4) and (2.14).

In a first subsection, we completely solve the problem in the easiest case where all the surface tensions are the same, and for any choice of a symmetric doublewell-shaped function $f$ satisfying (1.2).

In the second subsection, we address the general situation of different surface tension coefficients. This appears to be much more intricate and we are only able to conclude in some particular cases, for instance when $n \leqslant 4$, or by imposing slightly weaker consistency assumptions that appears to be sufficient in many cases, in particular from a numerical point of view. 


\subsection{Homogeneous surface tensions case}

We assume here that, for some $\sigma>0$, we have $\sigma_{i j}=\sigma, \forall i, j$. For the sake of simplicity, we replace everywhere it is possible the superscript $[\boldsymbol{\sigma}]$ by the superscript $[n]$ in this section. Indeed, it is only the number of components which is relevant here, and not the precise value of $\sigma$.

In this particular case, a straightforward computation shows that the coefficients $\overline{\boldsymbol{\alpha}}^{[\boldsymbol{\sigma}]}=\overline{\boldsymbol{\alpha}}^{[n]}$ defined in Lemma 2.1 are explicitly given by

$$
\begin{cases}\bar{\alpha}_{i j}^{[n]}=\frac{1}{n \sigma}, & \forall 1 \leqslant i \neq j \leqslant n, \\ \bar{\alpha}_{i i}^{[n]}=-(n-1) \frac{1}{n \sigma}, & \forall 1 \leqslant i \leqslant n .\end{cases}
$$

\subsubsection{Ansatz}

In order to find an expression for consistent potentials, we define a kind of basis of the space of all possible potentials, built upon the reference shape $f$ and chosen in such a way that its elements naturally satisfy some consistency properties.

More precisely, for all $1 \leqslant k \leqslant n$ we define the functions $\Psi_{k}^{[n]}[f]$ as follows

$$
\Psi_{k}^{[n]}[f](\mathbf{c}) \stackrel{\text { def }}{=} \sum_{\boldsymbol{i} \in I_{k}^{[n]}} \sum_{1 \leqslant s \leqslant k} \sum_{\boldsymbol{j} \in I_{s}^{[k]}(\boldsymbol{i})}(-1)^{k-s} f\left(c_{j_{1}}+\cdots+c_{j_{s}}\right), \quad \forall \mathbf{c} \in \mathbb{R}^{n},
$$

where the subsets $I_{k}^{[n]}(\boldsymbol{i})$ are defined for all $1 \leqslant k \leqslant n$ and for all $\boldsymbol{i}=\left(i_{1}, i_{2}, \ldots, i_{n}\right) \in$ $\mathbb{N}^{n}$ such that $i_{1}<i_{2}<\cdots<i_{n}$ as the subsets of all k-uplets of $\boldsymbol{i}$

$$
\left\{\begin{aligned}
I_{k}^{[n]}(\boldsymbol{i}) & =\left\{\left(i_{j_{1}}, i_{j_{2}}, \ldots, i_{j_{k}}\right), 1 \leqslant j_{1}<j_{2}<\cdots<j_{k} \leqslant n\right\} \\
I_{k}^{[n]} & =I_{k}^{[n]}(1,2, \ldots, n) .
\end{aligned}\right.
$$

In particular, it is worth noticing that using the assumptions (1.2), we have

$$
\begin{gathered}
\Psi_{n}^{[n]}[f](\mathbf{c})=f\left(c_{1}+\cdots+c_{n}\right)=f(1)=0, \quad \forall \mathbf{c} \in \mathcal{S}, \\
\Psi_{1}^{[n]}[f](\mathbf{c})=f\left(c_{1}\right)+\cdots+f\left(c_{n}\right), \quad \forall \mathbf{c} \in \mathcal{S} .
\end{gathered}
$$

It is convenient to express $\Psi_{k}^{[n]}[f]$ by means of the symmetric functions $S_{k}^{[n]}[f]$ defined as follows

$$
S_{k}^{[n]}[f](\mathbf{c}) \stackrel{\text { def }}{=} \sum_{\boldsymbol{i} \in I_{k}^{[n]}} f\left(c_{i_{1}}+c_{i_{2}}+\cdots+c_{i_{k}}\right), \forall \mathbf{c} \in \mathbb{R}^{n}, \forall 1 \leqslant k \leqslant n .
$$

By convention, we set $S_{0}^{[n]}[f](\mathbf{c}) \stackrel{\text { def }}{=} 0$ and we check that

$$
\Psi_{k}^{[n]}[f]=\sum_{1 \leqslant s \leqslant k}(-1)^{k-s}\left(\begin{array}{l}
n-s \\
k-s
\end{array}\right) S_{s}^{[n]}[f], \quad \forall 1 \leqslant k \leqslant n .
$$


We now look for $F^{[n]}$ as a linear combination of the functions $\left(\Psi_{k}^{[n]}[f]\right)_{1 \leqslant k \leqslant n}$

$$
F^{[n]}=\sum_{1 \leqslant k \leqslant n} \lambda_{k} \Psi_{k}^{[n]}[f]
$$

The coefficients $\left(\lambda_{k}\right)_{k \in \mathbb{N}^{*}}$ are real numbers that we need to determine. We do not add the superscript $n$ in this notation since we will see that eventually those coefficients will not depend on $n$. Observe that $F^{[n]}$ depends linearly on the reference two-phase potential $f$.

\subsubsection{Consistency Properties (2.3) and (2.4)}

As we said before, these functions $\Psi_{k}^{[n]}[f]$ are introduced because of their natural consistency properties that we state in the following proposition (proved in Appendix C)

Proposition 3.1. We have the following properties

$$
\begin{aligned}
\text { (i) } \Psi_{k}^{[n]}[f]\left(c_{1}, \ldots, c_{n-1}, 0\right) & =\Psi_{k}^{[n-1]}[f]\left(c_{1}, \ldots, c_{n-1}\right), \forall 1 \leqslant k<n, \\
\text { (ii) } \Psi_{n}^{[n]}[f]\left(c_{1}, \ldots, c_{n-1}, 0\right) & =0 .
\end{aligned}
$$

This proposition states that the functions $\left(\Psi_{k}^{[n]}[f]\right)_{1 \leqslant k \leqslant n}$ satisfy the Property (2.4) we want to impose to the potential $F^{[\boldsymbol{\sigma}]}=F^{[n]}$. Thus, by linearity, a potential $F^{[n]}$ defined by (3.3) automatically satisfies Property (2.4).

Moreover, a straightforward computation shows that Property (2.3) is equivalent to the condition

$$
2\left(\lambda_{1}-\lambda_{2}\right)=\sigma
$$

In the sequel, it remains to show that we can choose the coefficients $\lambda_{k}$ in order to ensure Property (2.14).

\subsubsection{Consistency Property (2.14)}

We recall that, in this section, we have assumed that all the surface tensions are the same. In this case, using (2.13) and (3.1), we see that the expression of the operators $L_{k}^{[\boldsymbol{\sigma}]}=L_{k}^{[n]}$, for $1 \leqslant k \leqslant n$ and for any smooth enough potential $F: \mathbb{R}^{n} \rightarrow \mathbb{R}$, reduces to

$$
L_{k}^{[n]}(F)=\frac{1}{n \sigma} \sum_{1 \leqslant s \leqslant n}\left(\partial_{k} F-\partial_{s} F\right) .
$$

By symmetry, we thus see that all the problem reduces to find values for the coefficients $\lambda_{k}, 1 \leqslant k \leqslant n$, such that the potential $F^{[n]}$ defined in (3.3) satisfies

$$
L_{n}^{[n]}\left(F^{[n]}\right)(\mathbf{c})=0, \text { for any } \mathbf{c} \in \mathcal{S} \text { such that } c_{n}=0 .
$$

The expression of $L_{n}^{[n]}\left(F^{[n]}\right)(\mathbf{c})$ is given in the following proposition (proved in Appendix D). 
Proposition 3.2. We have the following equality

$$
\begin{aligned}
L_{n}^{[n]}\left(F^{[n]}\right)\left(c_{1}, \ldots, c_{n-1}, 0\right) & \\
=-\frac{1}{n \sigma} \sum_{1 \leqslant k \leqslant n-2}\left[k \lambda_{k}+\right. & \left.\sum_{k+1 \leqslant s \leqslant n} \lambda_{s} \beta_{s, k}^{[n]}\right] S_{k}^{[n-1]}\left[f^{\prime}\right]\left(c_{1}, \ldots, c_{n-1}\right) \\
& +\frac{n-1}{n \sigma}\left(\lambda_{n}-\lambda_{n-1}\right) S_{n-1}^{[n-1]}\left[f^{\prime}\right]\left(c_{1}, \ldots, c_{n-1}\right),
\end{aligned}
$$

where the coefficients $\beta_{k, s}^{[n]}$ are defined as follows, for all $1 \leqslant s<n-1$ and $s<k \leqslant n$

$$
\beta_{k, s}^{[n]}=(-1)^{k-s}\left[\left(\begin{array}{l}
n-s-1 \\
k-s-1
\end{array}\right)(n-s-1)+\left(\begin{array}{l}
n-s \\
k-s
\end{array}\right) s\right] .
$$

Since, by (1.2), we have $S_{n-1}^{[n-1]}\left[f^{\prime}\right]\left(c_{1}, \ldots, c_{n-1}\right)=f^{\prime}\left(c_{1}+\cdots+c_{n-1}\right)=f^{\prime}(1)=0$ for any $\mathbf{c} \in \mathcal{S}$ such that $c_{n}=0,(3.4)$ is equivalent to the following set of equations

$$
k \lambda_{k}+\sum_{k+1 \leqslant s \leqslant n} \lambda_{s} \beta_{s, k}^{[n]}=0, \quad \forall 1 \leqslant k \leqslant n-2 .
$$

This is a maximal rank upper triangular linear system with $n-2$ equations and $n$ unknowns $\lambda_{1}, \ldots, \lambda_{n}$.

One can easily check that we have the following algebraic identities

$$
\begin{aligned}
& \text { (i) } \beta_{s, k+1}^{[n]}+\beta_{s, k}^{[n]}=\beta_{s, k}^{[n-1]}, \quad \forall 1 \leqslant k<n-2, \quad \forall k<s \leqslant n-1, \\
& \text { (ii) } \beta_{n, k}^{[n]}+\beta_{n, k+1}^{[n]}=0, \quad \forall 1 \leqslant k<n-2, \\
& \text { (iii) } \beta_{k+1, k}^{[n]}+k+1=\beta_{k+1, k}^{[n-1]}, \quad \forall 1 \leqslant k<n-2 .
\end{aligned}
$$

With those formulas at hand, the linear system (3.5) can be shown to be equivalent to the following maximal rank lower triangular system

$$
\lambda_{1}+\sum_{2 \leqslant s \leqslant k+1} \lambda_{s} \beta_{s, 1}^{[k+1]}=0, \quad \forall 2 \leqslant k \leqslant n-1 .
$$

Adding the constraint $2\left(\lambda_{1}-\lambda_{2}\right)=\sigma$ that we obtained in Section 3.1.2, one can easily check the following expression for the solutions of this system $\left(\lambda_{1}\right.$ being a free parameter)

$$
\lambda_{k}=\lambda_{1}-\frac{\sigma}{2} \sum_{s=1}^{k-1} \frac{1}{s}
$$

It is remarkable that such a simple explicit formula for the $\left(\lambda_{k}\right)_{k}$ can be obtained and, additionally, that this expression is independent of $n$ (and of $f$ ).

Remark 3.1. The value of $\lambda_{1}$ can be arbitrarily fixed. It has no influence on the potential $F^{[n]}$ since a straightforward computation and (1.2) shows that

$$
\sum_{1 \leqslant k \leqslant n} \Psi_{k}^{[n]}[f]=f\left(c_{1}+\cdots+c_{n}\right)=0, \quad \forall \mathbf{c} \in \mathcal{S} .
$$


In summary, we have proved that the potentials $F^{[n]}$ defined by (3.3) and (3.6) satisfy the three Properties (2.3), (2.4) and (2.14) (cf Section 3.1.3). Owing to the result stated in Section 2.5.2, we conclude that the family of models (2.12) associated with $F^{[n]}$ is a consistent hierarchy of Cahn-Hilliard systems (in the case where all the surface tension are the same).

\subsubsection{Example of the quartic potential $f(c)=c^{2}(1-c)^{2}$}

In practice, the Cahn-Hilliard equation is often considered with a reference polynomial potential of the following form

$$
f(c)=c^{2}(1-c)^{2} .
$$

In this case, the functions $\Psi_{k}^{[n]}[f]$ have a very simple structure. This is stated in the following result.

Proposition 3.3. Assume that $f$ is given by (3.7). For any $\mathbf{c} \in \mathcal{S}$, we have

$$
\begin{gathered}
\Psi_{1}^{[n]}[f](\mathbf{c})+\Psi_{2}^{[n]}[f](\mathbf{c})= \begin{cases}0 & \text { for } n=2 \text { or } n=3, \\
24 \sum_{i \in I_{4}^{[n]}} c_{i_{1}} c_{i_{2}} c_{i_{3}} c_{i_{4}} & \text { for } n \geqslant 4,\end{cases} \\
\Psi_{3}^{[n]}[f](\mathbf{c})= \begin{cases}0 & \text { for } n=3, \\
-48 \sum_{\boldsymbol{i} \in I_{4}^{[n]}} c_{i_{1}} c_{i_{2}} c_{i_{3}} c_{i_{4}} & \text { for } n \geqslant 4,\end{cases} \\
\Psi_{4}^{[n]}[f](\mathbf{c})=24 \sum_{\boldsymbol{i} \in I_{4}^{[n]}} c_{i_{1}} c_{i_{2}} c_{i_{3}} c_{i_{4}}, \quad \forall n \geqslant 4, \\
\Psi_{k}^{[n]}[f]=0, \quad \forall k \geqslant 5, \forall n \geqslant k .
\end{gathered}
$$

This leads to the following expression of the potential $F^{[n]}$

$$
\begin{gathered}
F^{[2]}(\mathbf{c})=\frac{\sigma}{2} \Psi_{1}^{[2]}[f](\mathbf{c})=\frac{\sigma}{2}\left(f\left(c_{1}\right)+f\left(c_{2}\right)\right)=\sigma f\left(c_{1}\right)=\sigma f\left(c_{2}\right), \quad \forall \mathbf{c} \in \mathcal{S}, \\
F^{[3]}(\mathbf{c})=\frac{\sigma}{2} \Psi_{1}^{[3]}[f](\mathbf{c})=\frac{\sigma}{2}\left(f\left(c_{1}\right)+f\left(c_{2}\right)+f\left(c_{3}\right)\right), \quad \forall \mathbf{c} \in \mathcal{S}, \\
F^{[n]}(\mathbf{c})=\frac{\sigma}{2} \Psi_{1}^{[n]}[f](\mathbf{c})+2 \sigma \sum_{\boldsymbol{i} \in I_{4}^{[n]}} c_{i_{1}} c_{i_{2}} c_{i_{3}} c_{i_{4}}, \\
=\frac{\sigma}{2}\left(\sum_{k=1}^{n} f\left(c_{k}\right)\right)+2 \sigma \sum_{i \in I_{4}^{[n]}} c_{i_{1}} c_{i_{2}} c_{i_{3}} c_{i_{4}}, \quad \forall \mathbf{c} \in \mathcal{S}, \forall n \geqslant 4 .
\end{gathered}
$$

In the case $n=3$, we recover the potential proposed in Ref. 3 , in the particular case of constant surface tensions which is considered here. 
To conclude this section, we are able to prove that in the case considered in this section, the $n$-phase potential that we propose is non-negative. This property (or at least a bound from below) is essential to ensure the well-posedness of the model (see Theorem 2.1).

Proposition 3.4. For all $\mathbf{c} \in \mathcal{S}$, for all $n \geqslant 2$, we have

$$
F^{[n]}(\mathbf{c}) \geqslant 0 .
$$

Proof. Since $f$ is non-negative, the cases $n=2$ and $n=3$ are straightforward by using the explicit formulas obtained above.

Assume now that $n \geqslant 4$. We introduce the Newton sums $\mathfrak{S}_{k}=\sum_{1 \leqslant i \leqslant n} c_{i}^{k}$. Thanks to the Newton identities, we find

$$
\sum_{i \in I_{4}^{[n]}} c_{i_{1}} c_{i_{2}} c_{i_{3}} c_{i_{4}}=\frac{1}{24}\left(1-6 \mathfrak{S}_{2}+3 \mathfrak{S}_{2}^{2}+8 \mathfrak{S}_{3}-6 \mathfrak{S}_{4}\right), \quad \forall \mathbf{c} \in \mathcal{S}
$$

and since $f(x)=x^{4}-2 x^{3}+x^{2}$,

$$
\Psi_{1}^{[n]}[f](\mathbf{c})=\mathfrak{S}_{4}-2 \mathfrak{S}_{3}+\mathfrak{S}_{2} .
$$

Thus, we have

$$
\begin{aligned}
F^{[n]} & =\frac{1}{24}\left(\left(12 \mathfrak{S}_{4}-24 \mathfrak{S}_{3}+12 \mathfrak{S}_{2}\right)+\left(2-12 \mathfrak{S}_{2}+6 \mathfrak{S}_{2}^{2}+16 \mathfrak{S}_{3}-12 \mathfrak{S}_{4}\right)\right) \\
& =\frac{1}{12}\left(1+3 \mathfrak{S}_{2}^{2}-4 \mathfrak{S}_{3}\right)=\frac{1}{12}\left(\left(1+\mathfrak{S}_{2}^{2}\right)-4 \mathfrak{S}_{3}+2 \mathfrak{S}_{2}^{2}\right) \\
& \geqslant \frac{1}{12}\left(2 \mathfrak{S}_{2}-4 \mathfrak{S}_{3}+2 \mathfrak{S}_{4}\right) \geqslant \frac{1}{6} \Psi_{1}^{[n]}[f] \geqslant 0, \quad \forall \mathbf{c} \in \mathcal{S} .
\end{aligned}
$$

It is worth noting that, in the particular case of homogeneous surface tensions, the potential $F^{[n]}$ satisfy the assumptions (2.22), and consequently that the wellposedness result stated in Theorem 2.1 holds.

\subsection{Arbitrary surface tensions case}

In the general case, it is not obvious to find explicit expressions of consistent $n$ phase Cahn-Hilliard potentials $F^{[\boldsymbol{\sigma}]}$. This is still an open problem and we will only give here some partial (yet useful) results in this direction.

\subsubsection{Consistency with 2-phase systems}

Mimicking the structure of the function $\Psi_{2}^{[n]}[f]$ introduced in the previous section, we define the following potential $F_{0}^{[\sigma]}$

$$
F_{0}^{[\boldsymbol{\sigma}]}(\mathbf{c})=\sum_{i<j} \frac{\sigma_{i j}}{2}\left[f\left(c_{i}\right)+f\left(c_{j}\right)-f\left(c_{i}+c_{j}\right)\right] .
$$


This potential satisfies Properties (2.3) and (2.4). Hence, we are now interested in checking Property (2.14). A straightforward computation shows that (for $1 \leqslant i \leqslant n$ )

$$
L_{i}^{[\boldsymbol{\sigma}]}\left(F_{0}^{[\boldsymbol{\sigma}]}\right)(\mathbf{c})=\sum_{1 \leqslant j, k \leqslant n} \frac{\bar{\alpha}_{i j}^{[\boldsymbol{\sigma}]} \sigma_{j k}}{2}\left[f^{\prime}\left(c_{j}\right)-f^{\prime}\left(c_{j}+c_{k}\right)\right] .
$$

In particular, for any two-phase state $\mathbf{c} \in \mathcal{S}$, that is such that $\widetilde{\mathbf{c}}^{\left\{j_{0}, k_{0}\right\}}=0$ for some $j_{0} \neq k_{0}$, we have

$$
L_{i}^{[\boldsymbol{\sigma}]}\left(F_{0}^{[\boldsymbol{\sigma}]}\right)(\mathbf{c})=0, \quad \forall i \notin\left\{j_{0}, k_{0}\right\} .
$$

Observe that this property holds for any choice of the double-well potential $f$.

This proves that the Cahn-Hilliard model associated with $F_{0}^{[\boldsymbol{\sigma}]}$ is consistent with all the underlying two-phase models for any two-phase potential $f$. It means that $\left(\mathcal{C}_{2}\right)$ and $\left(\mathcal{C}_{3}\right)$ are at least satisfied for $|I|=n-2$. This is clearly a weaker consistency requirement than the one expected. However, this is already an important property of the proposed models as mentionned for instance in Section 4 of Ref. 18. It ensures that any two-phase interface in the $n$-phase system will be properly captured.

\subsubsection{Consistency with 3-phase systems. The quartic polynomial case}

We restrict here the study to the case of the usual quartic two-phase potential $f(c)=c^{2}(1-c)^{2}$ (even though a similar analysis can be performed for higher order polynomial potentials). We are going to build, in that case, some $n$-phase CahnHilliard potentials which are consistent with the underlying 3 -phase systems. This means that $\left(\mathcal{C}_{2}\right)$ and $\left(\mathcal{C}_{3}\right)$ will be satisfied for $|I|=n-2$ but also for $|I|=n-3$. This new consistency property is thus stronger than the one discussed in the previous subsection and, from a modelling point of view, ensures that any triple point in the $n$-phase system will be properly captured.

The reason why we consider here the quartic potential is that it allows some simplifications in the sequel thanks to the following two identities available in that case

$$
\begin{gathered}
f^{\prime}(c+d)-f^{\prime}(c)-f^{\prime}(d)=-12 c d(1-c-d), \quad \forall c, d \in \mathbb{R}, \\
\sum_{1 \leqslant i \leqslant n} f^{\prime}\left(c_{i}\right)=12 \sum_{1 \leqslant j<k<l \leqslant n} c_{j} c_{k} c_{l}, \quad \forall \mathbf{c} \in \mathcal{S} .
\end{gathered}
$$

In particular with (3.10) we obtain, for all $\mathbf{c} \in \mathcal{S}$,

$$
\begin{aligned}
L_{i}^{[\boldsymbol{\sigma}]}\left(F_{0}^{[\boldsymbol{\sigma}]}\right)(\mathbf{c}) & =-\sum_{1 \leqslant j, k \leqslant n} \frac{\bar{\alpha}_{i j}^{[\boldsymbol{\sigma}]} \sigma_{j k}}{2}\left[f^{\prime}\left(c_{k}\right)-12 c_{j} c_{k}\left(1-c_{j}-c_{k}\right)\right] \\
& =-\sum_{1 \leqslant k \leqslant n} \frac{\left(\overline{\boldsymbol{\alpha}}^{[\boldsymbol{\sigma}]} \boldsymbol{\sigma}\right)_{i k}}{2} f^{\prime}\left(c_{k}\right)+6 \sum_{1 \leqslant j, k \leqslant n} \bar{\alpha}_{i j}^{[\boldsymbol{\sigma}]} \sigma_{j k} c_{j} c_{k} \sum_{\substack{1 \leqslant l \leqslant n \\
l \neq j, l \neq k}} c_{l} .
\end{aligned}
$$


Owing to (2.7), we get

$$
L_{i}^{[\boldsymbol{\sigma}]}\left(F_{0}^{[\boldsymbol{\sigma}]}\right)(\mathbf{c})=-\frac{1}{2} f^{\prime}\left(c_{i}\right)-\frac{\gamma_{i}}{2} \sum_{1 \leqslant k \leqslant n} f^{\prime}\left(c_{k}\right)+6 \sum_{\substack{1 \leqslant j, k, l \leqslant n \\ j \neq k, j \neq l, k \neq l}} \bar{\alpha}_{i j}^{[\boldsymbol{\sigma}]} \sigma_{j k} c_{j} c_{k} c_{l} .
$$

Finally, using (3.11), we arrive at the following identity

$$
L_{i}^{[\boldsymbol{\sigma}]}\left(F_{0}^{[\boldsymbol{\sigma}]}\right)(\mathbf{c})=-\frac{1}{2} f^{\prime}\left(c_{i}\right)-\sum_{1 \leqslant j<k<l \leqslant n} \Gamma_{i ;\{i, j, k, l\}}^{[\boldsymbol{\sigma}]} c_{j} c_{k} c_{l}, \forall \mathbf{c} \in \mathcal{S}
$$

with

$$
\Gamma_{i ;\{i, j, k, l\}}^{[\boldsymbol{\sigma}]} \stackrel{\text { def }}{=}-6\left[\bar{\alpha}_{i j}^{[\boldsymbol{\sigma}]}\left(\sigma_{j k}+\sigma_{j l}\right)+\bar{\alpha}_{i k}^{[\boldsymbol{\sigma}]}\left(\sigma_{j k}+\sigma_{k l}\right)+\bar{\alpha}_{i l}^{[\boldsymbol{\sigma}]}\left(\sigma_{j l}+\sigma_{k l}\right)-\gamma_{i}\right] .
$$

Let $\mathbf{c} \in \mathcal{S}$ such that $\widetilde{\mathbf{c}}^{\left\{j_{0}, k_{0}, l_{0}\right\}}=0$ for some $1 \leqslant j_{0}<k_{0}<l_{0} \leqslant n$, then we have

$$
L_{i}^{[\boldsymbol{\sigma}]}\left(F_{0}^{[\boldsymbol{\sigma}]}\right)(\mathbf{c})=-\Gamma_{i ;\left\{i, j_{0}, k_{0}, l_{0}\right\}}^{[\boldsymbol{\sigma}]} c_{j_{0}} c_{k_{0}} c_{l_{0}}, \quad \forall i \notin\left\{j_{0}, k_{0}, l_{0}\right\}
$$

The Cahn-Hilliard model defined from $F_{0}^{[\boldsymbol{\sigma}]}$ is thus not consistent with the underlying three-phase models. To compensate the term appearing in the right hand side of (3.12), we introduce a correction $G^{[\boldsymbol{\sigma}]}$ to the potential of the following form

$$
G^{[\boldsymbol{\sigma}]}(\mathbf{c}) \stackrel{\text { def }}{=} \sum_{\boldsymbol{i} \in I_{4}^{[n]}} \sum_{s \in \boldsymbol{i}} \Lambda_{s ; \boldsymbol{i}}^{[\boldsymbol{\sigma}]} H\left(c_{s}, \prod_{j \in \boldsymbol{i}} c_{j}\right) .
$$

where $\left(\Lambda_{s ; \boldsymbol{i}}^{[\boldsymbol{\sigma}]}\right)_{s, \boldsymbol{i}}$ are coefficients to be determined and $H$ is the continuous function defined by

$$
H(u, v) \stackrel{\text { def }}{=} \frac{|v| v}{|v|+u^{2}}, \forall(u, v) \neq(0,0), \quad \text { and } H(0,0) \stackrel{\text { def }}{=} 0 .
$$

Observe that $H$ is $\mathcal{C}^{1}$ on $\mathbb{R}^{2} \backslash\{(0,0)\}$ and its partial derivatives are given by

$$
\partial_{1} H(u, v)=\frac{-2 u|v| v}{\left(|v|+u^{2}\right)^{2}}, \text { and } \partial_{2} H(u, v)=\frac{2|v| u^{2}+v^{2}}{\left(|v|+u^{2}\right)^{2}}, \quad \forall(u, v) \neq(0,0) .
$$

Notice that $H$ is not differentiable at $(0,0)$ but its partial derivatives are given by

$$
\partial_{1} H(0,0)=0, \quad \partial_{2} H(0,0)=1 .
$$

With those properties, it is easy to check that the function $(\alpha, \beta) \mapsto \phi(\alpha, \beta)=$ $H(\alpha, \alpha \beta)$ is $\mathcal{C}^{1}$ on $\mathbb{R}^{2}$ and that we have

$$
\partial_{1} \phi(\alpha, \beta)=\partial_{1} H(\alpha, \alpha \beta)+\beta \partial_{2} H(\alpha, \alpha \beta) \text {, and } \partial_{2} \phi(\alpha, \beta)=\alpha \partial_{2} H(\alpha, \alpha \beta),
$$

even if the partial derivatives of $H$ are not continuous. Consequently, the function $G^{[\boldsymbol{\sigma}]}$ is itself of class $\mathcal{C}^{1}$ and its partial derivatives are given by

$$
\partial_{i} G^{[\boldsymbol{\sigma}]}(\mathbf{c})=\sum_{\substack{\boldsymbol{i} \in I_{4}^{[n]} \\ i \in \boldsymbol{i}}}\left(\Lambda_{i ; \boldsymbol{i}}^{[\boldsymbol{\sigma}]} \partial_{1} H\left(c_{i}, \prod_{j \in \boldsymbol{i}} c_{j}\right)+\sum_{s \in \boldsymbol{i}} \Lambda_{s ; \boldsymbol{i}}^{[\boldsymbol{\sigma}]} \partial_{2} H\left(c_{s}, \prod_{j \in \boldsymbol{i}} c_{j}\right) \prod_{\substack{j \in \boldsymbol{i} \\ j \neq i}} c_{j}\right)
$$


Let now $\mathbf{c} \in \mathcal{S}$ be a three-phase state, that is such that $\widetilde{\mathbf{c}}^{\left\{j_{0}, k_{0}, l_{0}\right\}}=0$ for some $1 \leqslant j_{0}<k_{0}<l_{0} \leqslant n$. Since at most 3 components of $\mathbf{c}$ are non zero, we know that all the products of four different components of $\mathbf{c}$ are zero. Using the properties of the partial derivatives of $H$, it remains that for such a $\mathbf{c}$, we have

$$
\partial_{i} G^{[\boldsymbol{\sigma}]}(\mathbf{c})=\sum_{\substack{\boldsymbol{i} \in I_{4}^{[n]} \\ i \in \boldsymbol{i}}}\left(\left(\sum_{s \in \boldsymbol{i}} \Lambda_{s ; i}^{[\boldsymbol{\sigma}]} \partial_{2} H\left(c_{s}, 0\right)\right) \prod_{\substack{j \in \boldsymbol{i} \\ j \neq i}} c_{j}\right) .
$$

- In the case where $i \in\left\{i_{0}, j_{0}, k_{0}\right\}$, we see that the three-term products $\prod_{\substack{j \in i \\ j \neq i}} c_{j}$ contains at least one term which is zero and therefore we have

$$
\partial_{i} G^{[\boldsymbol{\sigma}]}(\mathbf{c})=0, \forall i \in\left\{i_{0}, j_{0}, k_{0}\right\} .
$$

- If now $i \notin\left\{i_{0}, j_{0}, k_{0}\right\}$, the only three-term product which is possibly not zero, is the one where $\boldsymbol{i}=\left\{i, i_{0}, j_{0}, k_{0}\right\}$, and then

$$
\partial_{i} G^{[\boldsymbol{\sigma}]}(\mathbf{c})=\left(\sum_{s \in\left\{i, i_{0}, j_{0}, k_{0}\right\}} \Lambda_{s ; \boldsymbol{i}}^{[\boldsymbol{\sigma}]} \partial_{2} H\left(c_{s}, 0\right)\right) c_{i_{0}} c_{j_{0}} c_{k_{0}} .
$$

For $s=i$, we have $c_{s}=c_{i}=0$ and thus $\partial_{2} H\left(c_{s}, 0\right)=\partial_{2} H(0,0)=1$. For $s \in\left\{i_{0}, j_{0}, k_{0}\right\}$, the only case where $\partial_{2} H\left(c_{s}, 0\right)$ can be non zero is the one where $c_{s}=0$, but in that case, the product $c_{j_{0}} c_{k_{0}} c_{l_{0}}$ is zero. Finally, we have obtained

$$
\partial_{i} G^{[\boldsymbol{\sigma}]}(\mathbf{c})=\Lambda_{i ;\left\{i, i_{0}, j_{0}, k_{0}\right\}}^{[\boldsymbol{\sigma}]} c_{i_{0}} c_{j_{0}} c_{k_{0}}, \quad \forall i \notin\left\{i_{0}, j_{0}, k_{0}\right\} .
$$

This eventually leads to the formula

$$
L_{i}^{[\boldsymbol{\sigma}]}\left(G^{[\boldsymbol{\sigma}]}\right)(\mathbf{c})=\left(\sum_{\substack{1 \leqslant j \leqslant n \\ j \neq j_{0}, k_{0}, l_{0}}} \bar{\alpha}_{i j}^{[\boldsymbol{\sigma}]} \Lambda_{j ;\left\{j, j_{0}, k_{0}, l_{0}\right\}}^{[\boldsymbol{\sigma}]}\right) c_{j_{0}} c_{k_{0}} c_{l_{0}}
$$

Consequently, the correction potential $G^{[\boldsymbol{\sigma}]}$ will compensate (3.12), if and only if we can find coefficients $\Lambda_{j ;\left\{j, j_{0}, k_{0}, l_{0}\right\}}^{[\boldsymbol{\sigma}]}$ that satisfy the following linear system

$$
\sum_{\substack{1 \leqslant j \leqslant n \\ j \neq j_{0}, k_{0}, l_{0}}} \bar{\alpha}_{i j}^{[\boldsymbol{\sigma}]} \Lambda_{j ;\left\{j, j_{0}, k_{0}, l_{0}\right\}}^{[\boldsymbol{\sigma}]}=\Gamma_{i ;\left\{i, j_{0}, k_{0}, l_{0}\right\}}^{[\boldsymbol{\sigma}]}, \quad \forall i \in\{1, \ldots, n\} \backslash\left\{j_{0}, k_{0}, l_{0}\right\} .
$$

It appears that the existence and uniqueness of such coefficients $\Lambda_{\bullet ; \bullet}^{[\boldsymbol{\sigma}]}$ is ensured by the following lemma (whose proof is postponed to the Appendix B).

Lemma 3.1. The submatrix obtained from $\overline{\boldsymbol{\alpha}}^{[\boldsymbol{\sigma}]}$ by removing the same (non empty) set of rows and columns is invertible.

To summarize, we proved that the $n$-phase Cahn-Hilliard model defined from the potential $F_{0}^{[\boldsymbol{\sigma}]}+G^{[\boldsymbol{\sigma}]}$ is consistent with all the three-phase underlying models, if we define $G^{[\boldsymbol{\sigma}]}$ by (3.13), where the coefficients $\Lambda_{\bullet}^{[\boldsymbol{\sigma}] \boldsymbol{\bullet}}$ are the solutions of (3.14). 


\subsubsection{Stabilization of the potential}

At this point, we are not able to prove that the total consistent potential $F_{0}^{[\boldsymbol{\sigma}]}+G^{[\boldsymbol{\sigma}]}$ is a non-negative function (or even bounded from below). Actually, we know from Ref. 3 that, even for $n=3$, this might not be true and thus additional high order (polynomial) terms in the potential are needed to ensure the non-negativity of the three phase potential and thus the well-posedness of the system. We give here an extension of these terms to the $n$-phase case which allows to recover exactly the general form of the three-phase potential presented in Ref. 3. We refer to Section 4.3.2 for numerical results for which using such a stabilization procedure is mandatory. To achieve this goal, we define

$$
P^{[\boldsymbol{\sigma}]}(\mathbf{c}) \stackrel{\text { def }}{=} \sum_{1 \leqslant i<j<k \leqslant n} c_{i}^{2} c_{j}^{2} c_{k}^{2}-\sum_{1 \leqslant i<j \leqslant n} c_{i}^{2} c_{j}^{2} \sum_{\substack{1 \leqslant k<l \leqslant n \\ k \neq i, j \\ l \neq i, j}}\left(\Theta_{i j ; k ; l} H\left(c_{k}, c_{k} c_{l}\right)+\Theta_{i j ; l ; k} H\left(c_{l}, c_{k} c_{l}\right)\right),
$$

and the potential that we will eventually use, for some $\Lambda>0$, is

$$
F^{[\boldsymbol{\sigma}]}=F_{0}^{[\boldsymbol{\sigma}]}+G^{[\boldsymbol{\sigma}]}+\Lambda P^{[\boldsymbol{\sigma}]} .
$$

The role of the term $\Lambda P^{[\boldsymbol{\sigma}]}$ being only to enforce non-negativity of the total potential for $\Lambda>0$ large enough, we only need to check now that the coefficients $\Theta_{\bullet ; \bullet ; \bullet}$ can be chosen so as not to break the consistency properties of $F_{0}^{[\boldsymbol{\sigma}]}+G^{[\boldsymbol{\sigma}]}$.

By similar computations as in Section 3.2.2, we can obtain that

$$
\begin{aligned}
L_{i}^{[\boldsymbol{\sigma}]}\left(P^{[\boldsymbol{\sigma}]}\right)(\mathbf{c})= & \left(2 \bar{\alpha}_{i l_{0}}^{[\boldsymbol{\sigma}]}-\sum_{\substack{1 \leqslant j \leqslant n \\
j \neq j_{0}, k_{0}, l_{0}}} \bar{\alpha}_{i j}^{[\boldsymbol{\sigma}]} \Theta_{j_{0} k_{0} ; j ; l_{0}}\right) c_{j_{0}}^{2} c_{k_{0}}^{2} c_{l_{0}} \\
& +\left(2 \bar{\alpha}_{i k_{0}}^{[\boldsymbol{\sigma}]}-\sum_{\substack{1 \leqslant j \leqslant n \\
j \neq j_{0}, k_{0}, l_{0}}} \bar{\alpha}_{i j}^{[\boldsymbol{\sigma}]} \Theta_{j_{0} l_{0} ; j ; k_{0}}\right) c_{j_{0}}^{2} c_{k_{0}} c_{l_{0}}^{2} \\
& +\left(2 \bar{\alpha}_{i j_{0}}^{[\boldsymbol{\sigma}]}-\sum_{\substack{1 \leqslant j \leqslant n \\
j \neq j_{0}, k_{0}, l_{0}}} \bar{\alpha}_{i j}^{[\boldsymbol{\sigma}]} \Theta_{k_{0} l_{0} ; j ; j_{0}}\right) c_{j_{0}} c_{k_{0}}^{2} c_{l_{0}}^{2}
\end{aligned}
$$

We are thus led to impose, for any $1 \leqslant j_{0}<k_{0}<l_{0} \leqslant n$, the conditions

$$
\sum_{\substack{1 \leqslant j \leqslant n \\ \alpha \neq j_{0}, k_{0}, l_{0}}} \bar{\alpha}_{i j}^{[\boldsymbol{\sigma}]} \Theta_{j_{0} k_{0} ; j ; l_{0}}=2 \bar{\alpha}_{i l_{0}}^{[\boldsymbol{\sigma}]}, \quad \forall i \notin\left\{j_{0}, k_{0}, l_{0}\right\} .
$$

Those linear systems admit unique solutions thanks to Lemma 3.1. Observe that, in the case where $n=4$, we explicitely obtain

$$
\Theta_{i j ; k ; l}=\frac{2 \bar{\alpha}_{k l}^{[\sigma]}}{\bar{\alpha}_{k k}^{[\sigma]}}
$$




\section{Numerical examples}

Providing numerical simulations based on the $n$-phase Cahn-Hilliard model above is a challenging task and requires the development of specific numerical schemes (see Ref. 6 for the three phase model). This issue will be adressed in future work. Nevertheless, in this section, we propose several 2D numerical simulations based on the 3-phase and 4-phase Cahn-Hilliard models presented above. The aim is to illustrate the capabilities of such modelling.

The litterature about numerical simulations for Cahn-Hilliard equation is rather huge; the reader can refer to Ref. 23 (and reference therein) for a recent review.

In some numerical examples, the mobility $M_{\boldsymbol{\sigma}}$ is taken as a function (possibly degenerate) of the order parameters $\mathbf{c}$

$$
M_{\boldsymbol{\sigma}}(\mathbf{c})=M_{\mathrm{cst}}+M_{\mathrm{deg}} \prod_{i=1}^{n}\left(1-c_{i}\right)^{2}
$$

where $M_{\mathrm{cst}}, M_{\mathrm{deg}}$ are two non negative constants whose values will be given for each test case. It is easy to see that the consistency properties of the models are still valid in this case. Existence (in 2D and 3D) and uniqueness (in 2D) of weak solutions (see Theorem 2.1) also hold with a non-degenerate varying mobility, see Ref. 3.

\subsection{Coupling with Navier-Stokes equations}

The $n$-phase Cahn-Hilliard model can be coupled with the Navier-Stokes model (see Refs $1,2,5,21,27,22)$ as follows

- a transport term $\mathbf{u} \cdot \nabla c_{i}$ is added in the equation which describes the time evolution of the order parameter $c_{i}$,

- the density $\varrho$ and the viscosity $\eta$ are defined as positive, bounded and smooth function of the order parameters $\mathbf{c}$ whose values on single-phase states are given respectively by the physical values $\varrho_{i}$ and $\eta_{i}$ (for an explicit expression see e.g. Ref. 5).

- a capillary force term $\sum_{1 \leqslant i \leqslant n} \mu_{i} \nabla c_{i}$ is added in the right hand side of the momentum balance.

Since the density is defined as a function of order parameters, the mass balance is not satisfied in the interface between two phases. The conservative or non conservative forms of the Navier-Stokes equations do not allow to deduce a kinetic energy balance. This is the reason why we adopt the particular form of the Navier-Stokes equations below (the reader can find more details about this issue in Ref. 5). The 
$n$-phase Cahn-Hilliard/Navier-Stokes model we proposed reads as follows

$$
\begin{gathered}
\left\{\begin{array}{c}
\partial_{t} c_{i}+\mathbf{u} \cdot \nabla c_{i}=-\operatorname{div}\left(M_{\boldsymbol{\sigma}}(\mathbf{c}) \nabla\left(\sum_{j=1}^{n} \bar{\alpha}_{i j}^{[\boldsymbol{\sigma}]} \mu_{j}\right)\right), \quad 1 \leqslant i \leqslant n, \\
\mu_{i}=\frac{b}{\varepsilon} \partial_{i} F^{[\boldsymbol{\sigma}]}+\frac{a}{2} \varepsilon \sum_{1 \leqslant j \leqslant n} \sigma_{i j} \Delta c_{j}, \quad 1 \leqslant i \leqslant n, \\
\quad-\operatorname{div}(2 \eta(\mathbf{c}) D(\mathbf{u}))+\nabla p=\sum_{1 \leqslant i \leqslant n} \mu_{i} \nabla c_{i}+\varrho(\mathbf{c}) \mathbf{g}, \\
\operatorname{div} \mathbf{u}=0,
\end{array}\right.
\end{gathered}
$$

where $\mathbf{u}$ and $p$ stands for the velocity and the pressure of the mixture, $\mathbf{g}=$ $(0,0,-9.8)$ stands for the gravity.

We supplement the system with Neumann boundary conditions for the order parameters $c_{i}$ and for the chemical potentials $\mu_{i}$ (that is $\nabla c_{i} \cdot \mathbf{n}=0$ and $\nabla \mu_{i} \cdot \mathbf{n}=0$ ); and slip boundary conditions for the Navier-Stokes system (that is $\mathbf{u} \cdot \mathbf{n}=0$ and $[2 \eta D \mathbf{u} . \mathbf{n}-p \mathbf{n}] \cdot \boldsymbol{\tau}=0)$.

\subsection{Numerical schemes}

The space discretization is performed using a Galerkin formulation of the problem and the finite element method. We use a conformal $\mathbb{P}_{1}$ approximation for the order parameters $c_{i}$ and for the chemical potentials $\mu_{i}$. The velocity and the pressure are approximated using the $\mathbb{P}_{2} / \mathbb{P}_{1}$ inf-sup stable finite element. Moreover, we use an adaptive local refinement procedure (see Ref. 4) to refine the resolution of the discretization near the interfaces (so as to reach about 3 or 4 cells in the interfaces).

The time discretization consists in a splitting of Cahn-Hilliard and Navier-Stokes systems: we first solve the Cahn-Hilliard equations using the velocity computed at the previous time step in the transport term and then, we solve the Navier-Stokes equations using the updated order parameters and chemical potentials to build the capillary force term. For the Cahn-Hilliard step, we use a first order fully implicit Euler scheme. The resulting nonlinear discrete system is solved thanks to the Newton algorithm. For the Navier-Stokes equation, we use a semi-implicit scheme to linearize the discrete equation while preserving the discrete energy balance (the details of the discretization can be found in Ref. 5). The linear system is solved using an Uzawa method.

\subsection{Numerical simulations}

In complement of illustrations given in Ref. 3, we first compare, in Section 4.3.1, the results obtained with consistent and non-consistent three-phase potentials for the coupled Cahn-Hilliard/Navier-Stokes model. 
We then illustrate the behaviour of the 4-phase model in two different basic configurations:

- in Section 4.3.2, we solve the 4-phase Cahn-Hilliard system (without coupling with the Navier-Stokes equations), with different surface tensions but in a configuration such that no quadruple point appears. This illustrates the fact that the 4-phase model enables to simulate correctly two different three-phase systems by solving a single set of equations.

- in Section 4.3.3, we solve the coupled 4-phases Cahn-Hilliard/Navier-Stokes system with homogeneous surface tensions.

\subsubsection{Consistent model vs non-consistent model}

We present in this section the simulation of a rising bubble in a liquid under the effect of gravity. Only two phases are present but the simulation is performed using the 3-phase model. In this academic framework, we aim at illustrating the fact that the use of a model which is consistent or not can have an important influence on the velocity of the rising bubble since the representation of capillary forces may become incorrect due to the spurious appearance of the absent phase when using a non-consistent model. More precisely, we compare two simulations: the first one is performed using the potential $F_{0}^{[\boldsymbol{\sigma}]}$ (defined by (3.9)) which leads to a consistent model and the second one with the following potential (taken from Refs. 18, 24, 25) which leads to a non consistent model

$$
F_{0}^{\mathrm{nc}}\left(c_{1}, c_{2}, c_{3}\right)=\sigma_{12} c_{1}^{2} c_{2}^{2}+\sigma_{13} c_{1}^{2} c_{3}^{2}+\sigma_{23} c_{2}^{2} c_{3}^{2} .
$$

The 3D simulations are performed in cylindrical coordinates $(r, \theta, z)$ with an axisymmetry assumption for all the unknown of the problem. From a computational point of view, the simulation reduces to a $2 \mathrm{D}$ simulation in the $(r, z)$ plane. The computational domain is $[0,0.016] \times[0,0.12]$. The phase 1 represents the bubble, the phase 2 represents the liquid phase and the phase 3 is initially absent. At the initial time (see the left picture in Figure 1), the bubble is at rest and the order parameters are initialized by using the theoretical profile (A.2) for the variations in the transverse direction in the interfaces. The physical properties of the phases are given by the following parameters of the simulation

$$
\begin{aligned}
& \sigma_{12}=0.07, \sigma_{13}=0.07, \sigma_{23}=0.05, \\
& \varrho_{1}=1, \varrho_{2}=1000, \varrho_{3}=10000, \\
& \eta_{1}=10^{-4}, \eta_{2}=0.1, \eta_{3}=0.5 .
\end{aligned}
$$

The mobility is defined by (4.1) with $M_{\mathrm{cst}}=0$ and $M_{\mathrm{deg}}=5 \mathrm{e}-6$. The interface width is $\varepsilon=0.008$. The time step is equal to $10^{-3}$.

The position of the bubble during the time evolution using a consistent or a non consistent potential can be compared in Figure 1. The colors (in grayscale) represent the area where the order parameter $c_{1}$ is greater than 0.5 and the solid black lines 


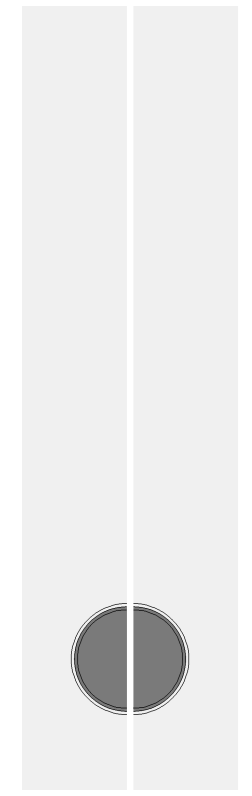

$\mathrm{t}=0$

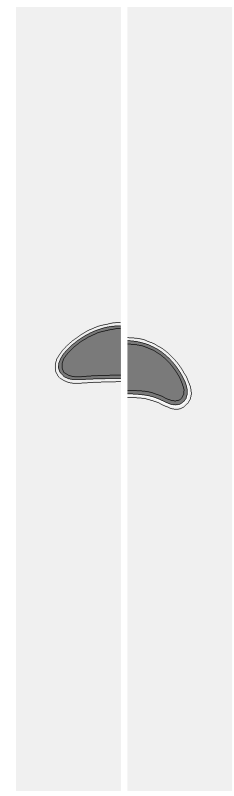

$\mathrm{t}=0.25$

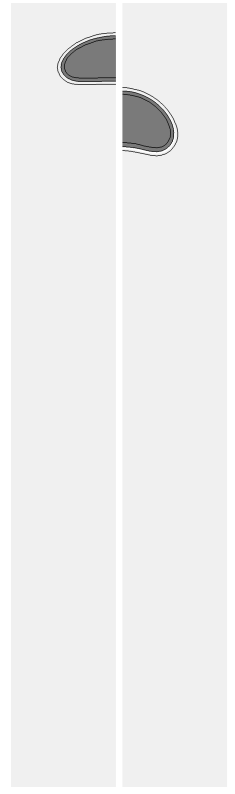

$\mathrm{t}=0.5$

Fig. 1: Rising of a single bubble: consistent (left) vs non consistent potential (right).

represent three contour lines of the order parameter $c_{1}$. We observe that the rising velocity of the bubble is more important when using the consistent potential. Figure 2 shows a vertical cutline $(\{r=0\})$ at time $t=0.5$ of order parameters $c_{1}$ and $c_{3}$. When using the non consistent model, the phase 3 appears in the interface between the phases 1 and 2, this is not the case when using the consistent model.

\subsubsection{Spreading of lens}

In this section, we present two test cases using the 4-phase Cahn-Hilliard model that we developped in this paper (and without coupling with Navier-Stokes equations here). The initial configuration (see Figure 3) is the same for both tests: two different lenses are placed between two other stratified phases.

The computational domain is $[-0.2,2.6] \times[-0.2,1.2]$ and $\varepsilon=0.08$ for both tests. The mobility is defined by (4.1) with $M_{\mathrm{cst}}=0.1$ and $M_{\mathrm{deg}}=10$.

For the first test case, the surface tensions are $\sigma_{12}=1, \sigma_{13}=0.8, \sigma_{14}=1.4$, $\sigma_{23}=1.1, \sigma_{24}=0.9, \sigma_{34}=1$. We use the potential $F_{0}^{[\boldsymbol{\sigma}]}+G^{[\boldsymbol{\sigma}]}$. The potential $F_{0}^{[\boldsymbol{\sigma}]}$ is defined by (3.9) and the potential $G^{[\boldsymbol{\sigma}]}$ by (3.13), the numerical values of coefficients involved in this definition being $\Lambda_{1 ;\{1,2,3,4\}}^{[\sigma]} \simeq 14.68, \Lambda_{2 ;\{1,2,3,4\}}^{[\sigma]} \simeq 15.13$, $\Lambda_{3 ;\{1,2,3,4\}}^{[\boldsymbol{\sigma}]} \simeq 15.14$ and $\Lambda_{4 ;\{1,2,3,4\}}^{[\boldsymbol{\sigma}]} \simeq 14.69$. A simplified expression of the partial 

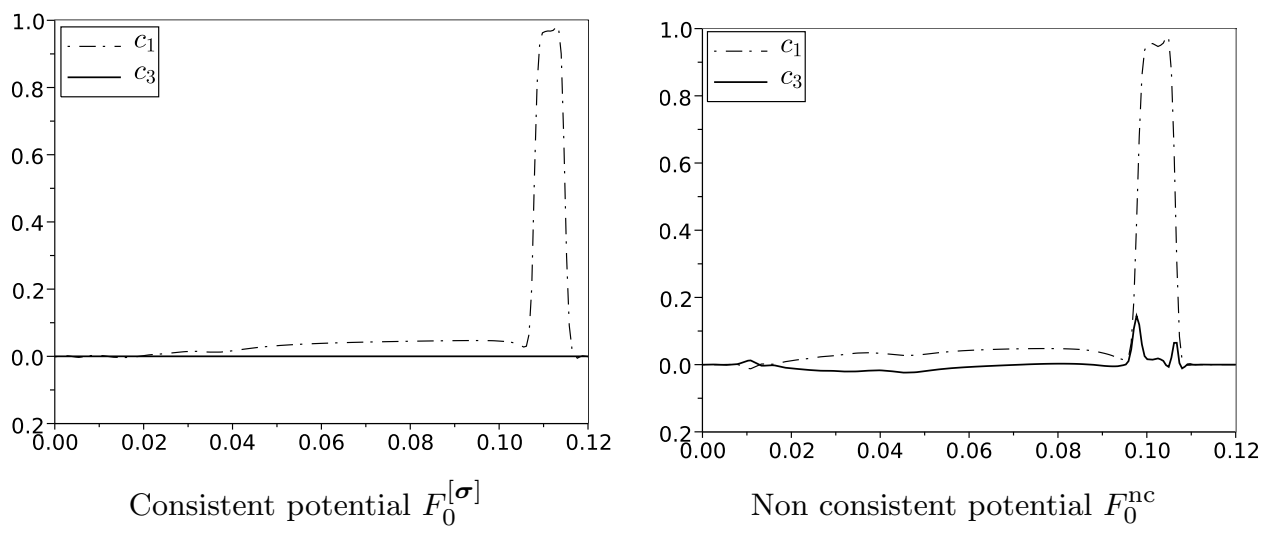

Fig. 2: Rising of a single bubble: vertical cutlines at $t=0.5$.

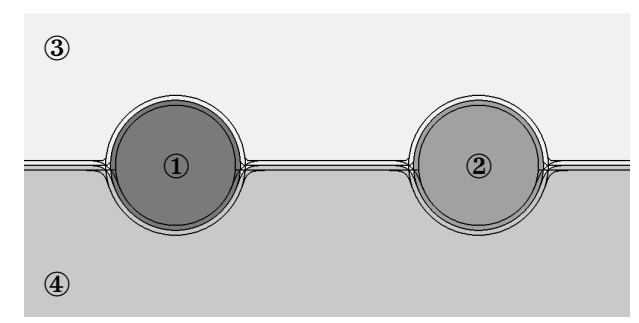

Fig. 3: Two lenses initial configuration and phase numbering

derivatives of $G^{[\boldsymbol{\sigma}]}$ (see Remark 4.1 below) can be used here since we a priori know that no quadruple point will appear during the simulation. The time step is equal to $10^{-4}$. The results we obtained at time $t=0.02$ and $t=1$ are presented in Figure 4. As previously, The colors (in grayscale) represent the area where the corresponding order parameter is greater than 0.5 and the solid black lines represent three contour lines of each order parameter. The two lenses partially spread between the two stratified phase and contact angles at triple points are consistent with the prescribed values of surface tensions (Young's relationship).

For the second test case, the surface tensions are $\sigma_{12}=1, \sigma_{13}=1, \sigma_{14}=$ $2.5, \sigma_{23}=2.5, \sigma_{24}=1, \sigma_{34}=1$. Note that these values do not satisfy the admissibility conditions presented in Section 2.3 for $n=4$. This is not crucial here since no quadruple point appears during the simulation and the admissibility conditions for $n=3$ holds for the four possible three-phase subsystems. We use the potential $F_{0}^{[\boldsymbol{\sigma}]}+G^{[\boldsymbol{\sigma}]}+\Lambda P^{[\boldsymbol{\sigma}]}$ with $\Lambda=21$. The potential $F_{0}^{[\boldsymbol{\sigma}]}$ is defined by (3.9), the potential $G^{[\boldsymbol{\sigma}]}$ by $(3.13)$ and the potential $P^{[\boldsymbol{\sigma}]}$ by $(3.15)$, the numerical values of coefficients involved in these definitions being $\Lambda_{i ;\{1,2,3,4\}}^{[\sigma]} \simeq 37.00$, for any 

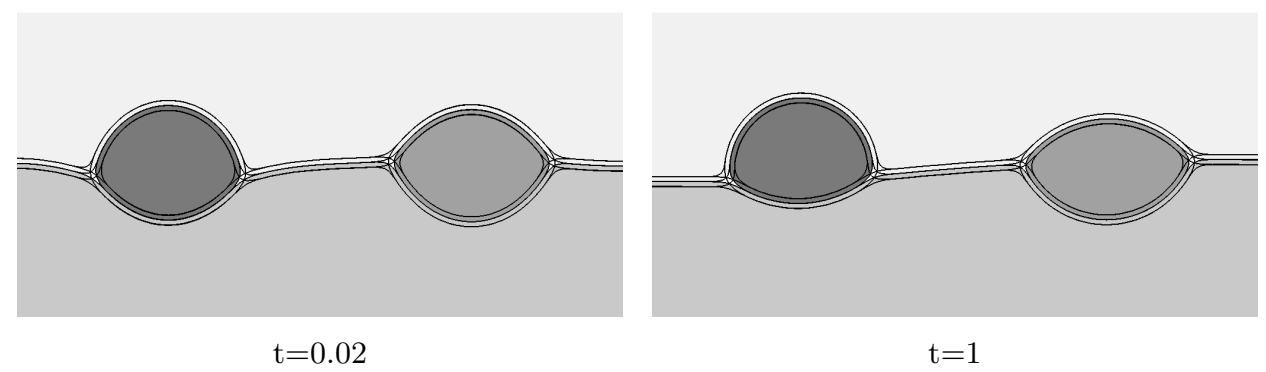

Fig. 4: Partial spreading of two lenses $\left(\sigma_{12}=1, \sigma_{13}=0.8, \sigma_{14}=1.4, \sigma_{23}=1.1\right.$, $\left.\sigma_{24}=0.9, \sigma_{34}=1\right)$.

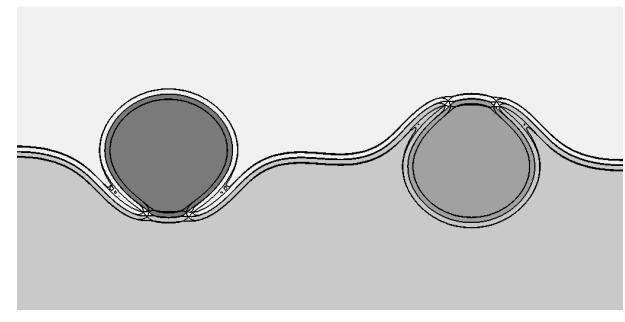

$t=0.008$

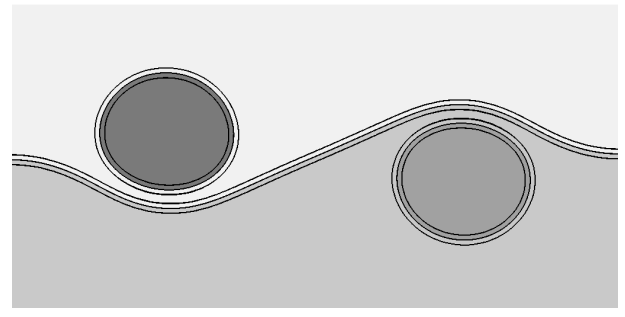

$t=0.1$

Fig. 5: Total spreading of two lenses

$\left(\sigma_{12}=1, \sigma_{13}=1, \sigma_{14}=2.5, \sigma_{23}=2.5, \sigma_{24}=1, \sigma_{34}=1\right)$.

$i=1,2,3,4$ and $\Theta_{k l ; i ; j}=\Theta_{k l ; j ; i}=-3.33$ if $(i, j) \in\{(1,2),(1,3),(2,4),(3,4)\}$, $\Theta_{k l ; i ; j}=\Theta_{k l ; j ; i}=4.67$ if $(i, j) \in\{(1,4),(2,3)\}$, with $\{i, j, k, l\}=\{1,2,3,4\}$. We use again a simplified expression of the partial derivatives of the potential $G^{[\sigma]}$ and $P^{[\sigma]}$ (see Remark 4.1 below). The time step is equal to $2 \mathrm{e}-5$. The results we obtained at time $t=0.008$ and $t=0.1$ are presented in Figure 5 . The two lenses are extracted from the interface between the two stratified phases. This physical motion corresponds to positive spreading coefficients.

Remark 4.1. It is interesting to note that the expressions of the derivatives of $G$ and $P^{[\sigma]}$ can be simplified at any point $\mathbf{c}$ which is a three-phase state. More precisely, for any $\mathbf{c} \in \mathcal{S}$ such that $\widetilde{\mathbf{c}}^{\left\{j_{0}, k_{0}, l_{0}\right\}}=0$, with $1 \leqslant j_{0}<j_{0}<k_{0} \leqslant n$, we can check that

$$
\begin{gathered}
\partial_{i} G^{[\boldsymbol{\sigma}]}(\mathbf{c})=\sum_{\substack{1 \leqslant j<k<l \leqslant n \\
j \neq i, k \neq i, l \neq i}} \Lambda_{i ;\{i, j, k, l\}}^{[\boldsymbol{\sigma}]} c_{j} c_{k} c_{l}, \quad \forall i \in\{1, \ldots, n\} . \\
\partial_{i} P^{[\boldsymbol{\sigma}]}(\mathbf{c})=2 c_{i} \sum_{1 \leqslant k<l \leqslant n} c_{k}^{2} c_{l}^{2}-\sum_{\substack{1 \leqslant j<k \leqslant n \\
j, k \neq i}} c_{j}^{2} c_{k}^{2} \sum_{\substack{1 \leqslant l \leqslant n \\
l \neq i, j, k}} \Theta_{j k ; i ; l} c_{l}, \quad \forall i \in\{1, \ldots, n\} .
\end{gathered}
$$




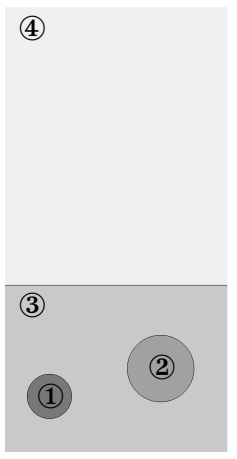

$t=0$

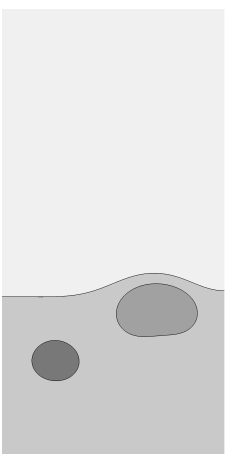

$t=0.08$

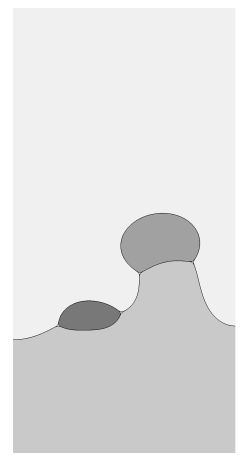

$t=0.16$

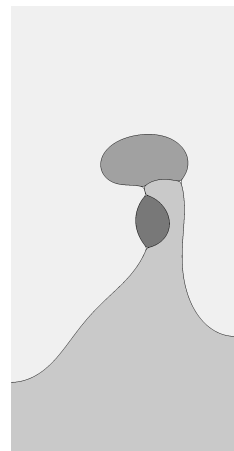

$t=0.24$

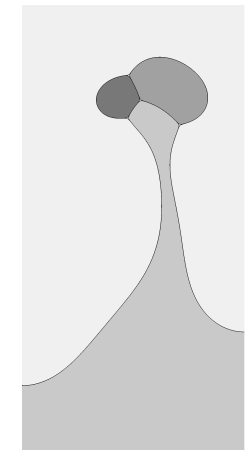

$t=0.32$

Fig. 6: Two bubbles rising through two statified phases $\left(\sigma_{i j}=1, \forall i, j\right)$

This gives a simple smooth expression of the partial derivatives of $G^{[\boldsymbol{\sigma}]}$ and $P^{[\boldsymbol{\sigma}]}$ which is valid in the absence of quadruple point in the system. This is in particular useful when implementing numerical schemes for this problem.

\subsubsection{Rising bubbles across interfaces}

In this section, we present two examples of simulations using the 4-phase CahnHilliard/Navier-Stokes model. All the surface tensions are equal to 0.05. We use the potential $F^{[4]}$ defined by (3.8).

The first test case corresponds to two bubbles (made of different phases), initially at rest, rising in a mixture of two stratified heavier phases under the effect of gravity. The time step is equal to $5 \mathrm{e}-4$. The computational domain is $[0,0.4] \times[0,0.8]$ and the initial configuration is shown on the left picture of Figure 6 . The densities and viscosities in pure phases are

$$
\begin{aligned}
& \varrho_{1}=1, \varrho_{2}=0.178, \varrho_{3}=1200, \varrho_{4}=1000 \\
& \eta_{1}=1 \mathrm{e}-4, \eta_{2}=1 \mathrm{e}-4, \eta_{3}=0.15, \eta_{4}=0.1 .
\end{aligned}
$$

The mobility is defined by (4.1) with $M_{\mathrm{cst}}=0$ and $M_{\mathrm{deg}}=5 \mathrm{e}-2$.

Figure 6 presents the time evolution of the system we obtain. The colors (in grayscale) represent the area where the corresponding order parameter is greater than 0.5 and the solid black lines represent the contour lines $c_{i}=0.5$ of each order parameter.

The second test case corresponds to a single bubble, initially at rest, rising in a mixture of three stratified heavier phases under the effect of gravity. The time step is equal to $5 \mathrm{e}-4$. The computational domain is $[-1.2 \mathrm{e}-2,1.2 \mathrm{e}-2] \times[0,1.44 \mathrm{e}-1]$ and the initial configuration is shown on the left picture of Figure 7 . The densities 


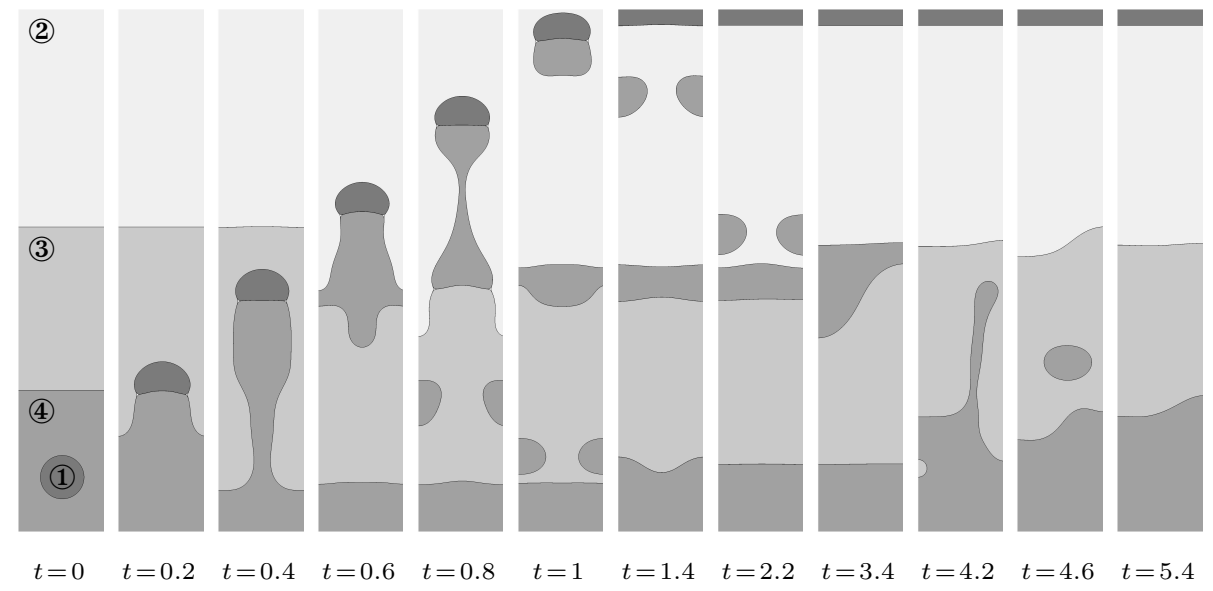

Fig. 7: One bubble rising through three statified phases $\left(\sigma_{i j}=1, \forall i, j\right)$

and viscosities in pure phases are

$$
\begin{aligned}
& \varrho_{1}=1, \varrho_{2}=1000, \varrho_{3}=1100, \varrho_{4}=1200, \\
& \eta_{1}=1 . \mathrm{e}-4, \eta_{2}=0.1, \eta_{3}=0.01, \eta_{4}=0.001 .
\end{aligned}
$$

The mobility is defined by (4.1) with $M_{\text {cst }}=0$ and $M_{\mathrm{deg}}=1 \mathrm{e}-2$.

Figure 7 presents the time evolution of the system we obtain and Figure 8 shows 20 contour lines of order parameters at $t=1$. We observe that no spurious phases appears during the simulation showing that the model is able to handle quite complex situations while capturing correctly all the interfaces during the computation.

\section{Appendices}

\section{A. Coefficients of the two-phase free energy}

We need to connect the parameters $A$ and $B$ in the definition (1.1) of $\mathcal{F}$ with the two physically and numerically relevant quantities: surface tension and interface thickness. To this end, we analyse the case of a single interface on the whole real line, which amounts at minimising the energy

$$
c \in H_{l o c}^{1}(\mathbb{R}) \mapsto \mathcal{F}_{1 \mathrm{D}}(c)=\int_{\mathbb{R}} \frac{A}{2}\left|c^{\prime}(x)\right|^{2}+B f(c(x)) d x,
$$

among all the functions connecting 0 to 1 , that is such that $\lim _{-\infty} c=0$ and $\lim _{+\infty} c=1$. Easy calculations lead to the following differential equation for the minimiser $c_{A, B}$

$$
-A c_{A, B}^{\prime \prime}+B f^{\prime}\left(c_{A, B}\right)=0,
$$

and then, since $f(0)=f(1)=0$, to the equation

$$
\frac{A}{2}\left|c_{A, B}^{\prime}(x)\right|^{2}=B f\left(c_{A, B}(x)\right), \quad \forall x \in \mathbb{R} \text {. }
$$




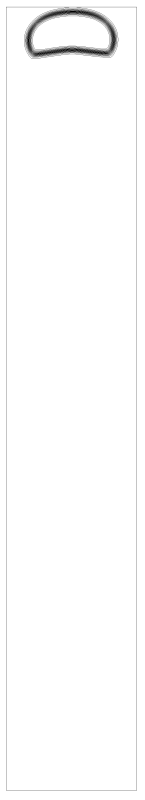

$c_{1}$

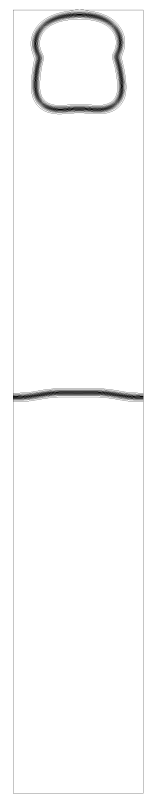

$c_{2}$

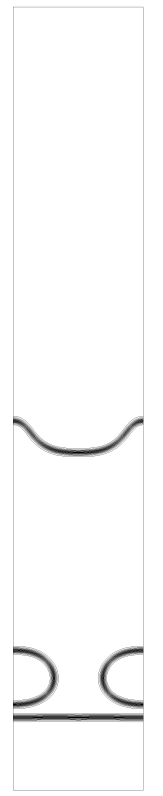

$c_{3}$

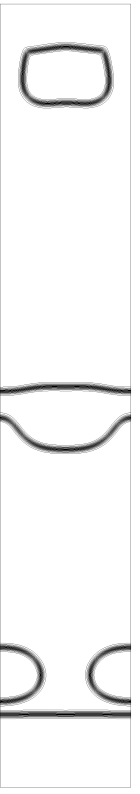

$c_{4}$

Fig. 8: One bubble rising through three statified phases $\left(\sigma_{i j}=1, \forall i, j\right)$, 20 contour lines of order parameters at $t=1$.

This imply that $0<c_{A, B}(x)<1$ and $c_{A, B}^{\prime}(x)>0$ for any $x \in \mathbb{R}$, so that we get

$$
c_{A, B}^{\prime}(x)=\sqrt{\frac{2 B}{A} f\left(c_{A, B}(x)\right)} .
$$

The function $c_{A, B}$, which is unique up to translations, corresponds to the interface profile and only depends on the ratio $B / A$. We decide to define the interface thickness $\varepsilon$ by the following formula

$$
\varepsilon=\frac{1}{\sup c_{A, B}^{\prime}}=\sqrt{\frac{A}{2 B \max _{[0,1]} f}},
$$

whose meaning is illustrated in Figure 9.

Moreover, in such a model the surface tension is defined as the total excess energy related to the concentration variation in the interface that is, since $f(0)=f(1)=0$,

$$
\sigma=\mathcal{F}_{1 \mathrm{D}}\left(c_{A, B}\right)=\int_{\mathbb{R}} \frac{A}{2}\left|c_{A, B}^{\prime}(x)\right|^{2}+B f\left(c_{A, B}(x)\right) d x .
$$

From (A.1), we see that the two terms in the integral are actually equal, so that a simple change of variables in the integral gives

$$
\sigma=A \int_{\mathbb{R}}\left|c_{A, B}^{\prime}(x)\right|^{2} d x=\sqrt{2 A B} \int_{\mathbb{R}} \sqrt{f\left(c_{A, B}(x)\right)} c_{A, B}^{\prime}(x) d x=\sqrt{2 A B} \int_{0}^{1} \sqrt{f} .
$$




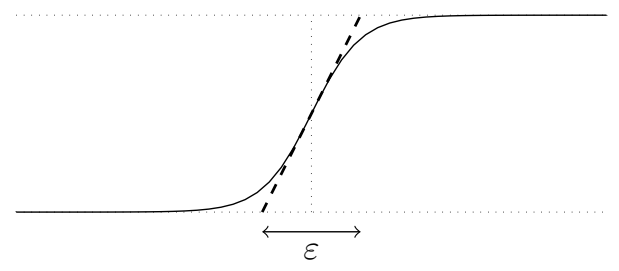

Fig. 9: Typical interface profile; Interface width

Combining the previous computations, we find the expressions (1.4) of $A$ and $B$.

Remark A.1. The very classical case is to consider the fourth-order polynomial potential $f(c)=c^{2}(1-c)^{2}$, in which case we find $a=\frac{3}{2}, \quad b=12$, and

$$
c_{A, B}(x)=\frac{1}{2}\left(1+\tanh \left(\frac{2 x}{\varepsilon}\right)\right) \text {, }
$$

as in Refs. 3, 5 .

\section{B. Proofs of technical results about the matrix $\bar{\alpha}^{[\sigma]}$}

We give in this section the proofs of the two technical lemmas 2.1 and 2.2 which concern existence, uniqueness and main properties of the matrix $\overline{\boldsymbol{\alpha}}^{[\boldsymbol{\sigma}]}$.

\section{Proof (of Lemma 2.1).}

- For any $1 \leqslant i \leqslant n$, we look for a row vector $\boldsymbol{\alpha}_{i} \in M_{1, n}(\mathbb{R})$ and two real numbers $\beta_{i}, \gamma_{i} \in \mathbb{R}$ satisfying

$$
\left\{\begin{array}{l}
\boldsymbol{\alpha}_{i} \boldsymbol{\sigma}=\beta_{i}{ }^{t} \boldsymbol{e}_{i}+\gamma_{i}{ }^{t} \mathbf{1}, \\
\boldsymbol{\alpha}_{i} \mathbf{1}=0
\end{array}\right.
$$

Observe that (B.1) is a system of $n+1$ equations with $n+2$ unknowns so that it admits at least one non trivial solution.

Let us show that $\beta_{i} \neq 0$. Indeed, if we assume that $\beta_{i}=0$ we have

$$
\boldsymbol{\alpha}_{i} \boldsymbol{\sigma}=\gamma_{i}{ }^{t} \mathbf{1}
$$

and thus

$$
\boldsymbol{\alpha}_{i} \boldsymbol{\sigma}^{t} \boldsymbol{\alpha}_{i}=\gamma_{i}{ }^{t} \mathbf{1}^{t} \boldsymbol{\alpha}_{i}=\gamma_{i}{ }^{t}\left(\boldsymbol{\alpha}_{i} \mathbf{1}\right)=0 .
$$

Since $\boldsymbol{\alpha}_{i} \mathbf{1}=0$, we deduce from (2.6) that $\boldsymbol{\alpha}_{i}=0$. Coming back to (B.1), we conclude that finally $\beta_{i}=\gamma_{i}=0$ and $\boldsymbol{\alpha}_{i}=0$ which is not possible since we considered a non trivial solution of (B.1).

This also proves that the space of solutions of (B.1) is of dimension 1 .

- We can now define the matrix $\overline{\boldsymbol{\alpha}}^{[\boldsymbol{\sigma}]}$ whose rows are given by $\boldsymbol{\alpha}_{i} / \beta_{i}$ and the vector $\boldsymbol{\gamma}^{[\boldsymbol{\sigma}]}=\left(\gamma_{i} / \beta_{i}\right)_{1 \leqslant i \leqslant n}$, in order to obtain the unique solution to

$$
\overline{\boldsymbol{\alpha}}^{[\boldsymbol{\sigma}]} \boldsymbol{\sigma}=\mathrm{Id}+\boldsymbol{\gamma}^{[\boldsymbol{\sigma}]} \otimes \mathbf{1}
$$




$$
\overline{\boldsymbol{\alpha}}^{[\sigma]} \mathbf{1}=0 .
$$

- To prove that $\overline{\boldsymbol{\alpha}}^{[\boldsymbol{\sigma}]}$ is symmetric, we right-multiply the matrix equation by ${ }^{t}\left(\overline{\boldsymbol{\alpha}}^{[\boldsymbol{\sigma}]}\right)$ to get

$$
\overline{\boldsymbol{\alpha}}^{[\boldsymbol{\sigma}]} \boldsymbol{\sigma}^{t}\left(\overline{\boldsymbol{\alpha}}^{[\boldsymbol{\sigma}]}\right)={ }^{t}\left(\overline{\boldsymbol{\alpha}}^{[\boldsymbol{\sigma}]}\right)+\boldsymbol{\gamma}^{[\boldsymbol{\sigma}]} \otimes \mathbf{1}^{t}\left(\overline{\boldsymbol{\alpha}}^{[\boldsymbol{\sigma}]}\right)={ }^{t}\left(\overline{\boldsymbol{\alpha}}^{[\boldsymbol{\sigma}]}\right)+\boldsymbol{\gamma}^{[\boldsymbol{\sigma}]} \otimes \underbrace{t}_{=0} \underbrace{\left(\overline{\boldsymbol{\alpha}}^{[\boldsymbol{\sigma}]} \mathbf{1}\right)} .
$$

Since $\boldsymbol{\sigma}$ is symmetric, we conclude that $\overline{\boldsymbol{\alpha}}^{[\boldsymbol{\sigma}]}$ is also symmetric and that the following relation holds

$$
\overline{\boldsymbol{\alpha}}^{[\boldsymbol{\sigma}]} \boldsymbol{\sigma} \overline{\boldsymbol{\alpha}}^{[\boldsymbol{\sigma}]}=\overline{\boldsymbol{\alpha}}^{[\boldsymbol{\sigma}]} .
$$

- Finally, we consider $\boldsymbol{\xi} \in \operatorname{Ker} \overline{\boldsymbol{\alpha}}^{[\boldsymbol{\sigma}]}$ and we right multiply the transpose of the matrix equation by $\boldsymbol{\xi}$ to obtain

$$
\boldsymbol{\sigma} \overline{\boldsymbol{\alpha}}^{[\sigma]} \boldsymbol{\xi}=\boldsymbol{\xi}+\left(\boldsymbol{\gamma}^{[\boldsymbol{\sigma}]} \cdot \boldsymbol{\xi}\right) \mathbf{1}
$$

Since $\boldsymbol{\xi} \in \operatorname{Ker} \overline{\boldsymbol{\alpha}}^{[\boldsymbol{\sigma}]}$, we get

$$
\boldsymbol{\xi}=-\left(\gamma^{[\sigma]} \cdot \boldsymbol{\xi}\right) \mathbf{1}
$$

which proves that $\boldsymbol{\xi} \in \operatorname{Span}\{\mathbf{1}\}$. This concludes the proof of the lemma.

Proof (of Lemma 2.2). We use (2.8) to deduce that

$$
\bar{\alpha}_{l l}^{[\sigma]}={ }^{t} e_{l} \overline{\boldsymbol{\alpha}}^{[\sigma]} \boldsymbol{\sigma} \overline{\boldsymbol{\alpha}}^{[\boldsymbol{\sigma}]} \boldsymbol{e}_{l} .
$$

Moreover, since $\overline{\boldsymbol{\alpha}}^{[\boldsymbol{\sigma}]} \boldsymbol{e}_{l} \cdot \mathbf{1}=0$ and $\overline{\boldsymbol{\alpha}}^{[\boldsymbol{\sigma}]} \boldsymbol{e}_{l}$ is not zero (see (1.10)), we can use (2.6) to obtain that $\bar{\alpha}_{l l}^{[\boldsymbol{\sigma}]} \neq 0$. Without loss of generality, we prove the equality (2.15) only for $l=n$. Since the matrix $\overline{\boldsymbol{\alpha}}^{[\boldsymbol{\sigma}]}$ is uniquely defined by (2.7) and symmetric, we can compute the following quantities, for $1 \leqslant i, j<n$,

$$
\left\{\begin{aligned}
\sum_{k=1}^{n-1}\left[\bar{\alpha}_{i k}^{[\boldsymbol{\sigma}]}-\frac{\left.\bar{\alpha}_{i n}^{[\boldsymbol{\sigma}]} \bar{\alpha}_{k n}^{[\boldsymbol{\sigma}]}\right]}{\bar{\alpha}_{n n}^{[\boldsymbol{\sigma}]}}\right] \sigma_{k j} & =\left(\overline{\boldsymbol{\alpha}}^{[\boldsymbol{\sigma}]} \boldsymbol{\sigma}\right)_{i j}-\frac{\bar{\alpha}_{i n}^{[\boldsymbol{\sigma}]}}{\bar{\alpha}_{n n}^{[\boldsymbol{\sigma}]}}\left(\overline{\boldsymbol{\alpha}}^{[\boldsymbol{\sigma}]} \boldsymbol{\sigma}\right)_{n j} \\
& =\delta_{i j}+\gamma_{i}^{[\boldsymbol{\sigma}]}-\frac{\bar{\alpha}_{i n}^{[\boldsymbol{\sigma}]}}{\bar{\alpha}_{n n}^{[\boldsymbol{\sigma}]}} \gamma_{n}^{[\boldsymbol{\sigma}]}, \\
\sum_{k=1}^{n-1}\left(\bar{\alpha}_{i k}^{[\boldsymbol{\sigma}]}-\frac{\bar{\alpha}_{i n}^{[\boldsymbol{\sigma}]} \bar{\alpha}_{k n}^{[\boldsymbol{\sigma}]}}{\bar{\alpha}_{n n}^{[\boldsymbol{\sigma}]}}\right) & =0 .
\end{aligned}\right.
$$

This proves that the coefficients defined by the right-hand side of (2.15) satisfy the relationship (2.7) with $\boldsymbol{\gamma}^{\left[\widetilde{\boldsymbol{\sigma}}^{n}\right]}$ defined by

$$
\gamma_{i}^{\left[\widetilde{\boldsymbol{\sigma}}^{n}\right]}=\gamma_{i}^{[\boldsymbol{\sigma}]}-\frac{\bar{\alpha}_{i n}^{[\boldsymbol{\sigma}]}}{\bar{\alpha}_{n n}^{[\boldsymbol{\sigma}]}} \gamma_{n}^{[\boldsymbol{\sigma}]}, \quad i=1, \ldots, n-1 .
$$

The uniqueness stated in Lemma 2.1 proves the result. 
Proof (of Lemma 3.1). To simplify the notation in the proof, we set $\boldsymbol{\alpha}=\overline{\boldsymbol{\alpha}}^{[\boldsymbol{\sigma}]}$, $\gamma=\gamma^{[\boldsymbol{\sigma}]}$. We write $\boldsymbol{\alpha}$ in the following block form

$$
\boldsymbol{\alpha}=\left(\begin{array}{cc}
\tilde{\boldsymbol{\alpha}} & \boldsymbol{\delta} \\
{ }^{t} \boldsymbol{\delta} & \hat{\boldsymbol{\alpha}}
\end{array}\right)
$$

and accordingly

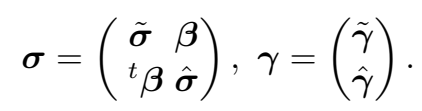

Since the indexes of rows and columns can be permuted, it is sufficient to prove that that the submatrix $\hat{\boldsymbol{\alpha}}$ is invertible. The equation (2.11) leads to

$$
\begin{gathered}
\tilde{\boldsymbol{\alpha}} \tilde{\boldsymbol{\sigma}}+\boldsymbol{\delta}^{t} \boldsymbol{\beta}=\tilde{\mathrm{Id}}+\tilde{\boldsymbol{\gamma}} \otimes \tilde{\mathbf{1}}, \\
{ }^{t} \boldsymbol{\delta} \tilde{\boldsymbol{\sigma}}+\hat{\boldsymbol{\alpha}}{ }^{t} \boldsymbol{\beta}=\hat{\boldsymbol{\gamma}} \otimes \tilde{\mathbf{1}} \\
{ }^{t} \boldsymbol{\delta} \boldsymbol{\beta}+\hat{\boldsymbol{\alpha}} \hat{\boldsymbol{\sigma}}=\hat{\mathrm{Id}}+\hat{\boldsymbol{\gamma}} \otimes \hat{\mathbf{1}} .
\end{gathered}
$$

Let us consider a $\hat{\boldsymbol{\xi}}$ such that $\hat{\boldsymbol{\alpha}} \hat{\boldsymbol{\xi}}=0$. Since ${ }^{t} \mathbf{1} \boldsymbol{\alpha}=0$ we have

$$
0={ }^{t} \mathbf{1} \boldsymbol{\alpha}\left(\begin{array}{l}
0 \\
\hat{\boldsymbol{\xi}}
\end{array}\right)={ }^{t} \mathbf{1}\left(\begin{array}{c}
\boldsymbol{\delta} \hat{\boldsymbol{\xi}} \\
\hat{\boldsymbol{\alpha}} \hat{\boldsymbol{\xi}}
\end{array}\right)={ }^{t} \tilde{\mathbf{1}} \boldsymbol{\delta} \hat{\boldsymbol{\xi}}
$$

and thus

$$
\tilde{\mathbf{1}} \cdot(\boldsymbol{\delta} \hat{\boldsymbol{\xi}})=0
$$

Moreover, from (B.3), we have

$$
\tilde{\boldsymbol{\sigma}} \boldsymbol{\delta} \hat{\boldsymbol{\xi}}+\underbrace{\boldsymbol{\beta} \hat{\boldsymbol{\alpha}} \hat{\boldsymbol{\xi}}}_{=0}=(\hat{\gamma} \cdot \hat{\boldsymbol{\xi}}) \tilde{\mathbf{1}} .
$$

We deduce, using (B.5) that

$$
{ }^{t}(\boldsymbol{\delta} \hat{\boldsymbol{\xi}}) \tilde{\boldsymbol{\sigma}}(\boldsymbol{\delta} \hat{\boldsymbol{\xi}})=0,
$$

and from (2.6) (which is also satisfied by the submatrix $\tilde{\sigma}$ ), we deduce that

$$
\delta \hat{\boldsymbol{\xi}}=0 .
$$

Bearing in mind (B.6), we get

$$
(\hat{\boldsymbol{\xi}} \cdot \hat{\gamma})=0
$$

We now multiply the transpose of (B.4) by $\hat{\xi}$ we get

$$
\hat{\boldsymbol{\xi}}+\underbrace{(\hat{\gamma} \cdot \hat{\boldsymbol{\xi}})}_{=0} \hat{\mathbf{1}}={ }^{t} \boldsymbol{\beta} \underbrace{\boldsymbol{\delta} \hat{\boldsymbol{\xi}}}_{=0}+\hat{\boldsymbol{\sigma}} \underbrace{\hat{\alpha} \hat{\boldsymbol{\xi}}}_{=0}=0 .
$$

We get that $\hat{\boldsymbol{\xi}}=0$. This proves that $\hat{\boldsymbol{\alpha}}$ is invertible. 


\section{Proof of proposition 3.1}

We use the equality (3.2) to prove Proposition 3.1 and to this end, we begin with the following lemma

Lemma C.1. We have the following equalities (for all $1<k<n$ )

(i) $S_{1}^{[n]}[f]\left(c_{1}, \ldots, c_{n-1}, 0\right)=S_{1}^{[n-1]}[f]\left(c_{1}, \ldots, c_{n-1}\right)$,

(ii) $S_{k}^{[n]}[f]\left(c_{1}, \ldots, c_{n-1}, 0\right)=S_{k-1}^{[n-1]}[f]\left(c_{1}, \ldots, c_{n-1}\right)+S_{k}^{[n-1]}[f]\left(c_{1}, \ldots, c_{n-1}\right)$,

(iii) $S_{n}^{[n]}[f]\left(c_{1}, \ldots, c_{n-1}, 0\right)=S_{n-1}^{[n-1]}[f]\left(c_{1}, \ldots, c_{n-1}\right)$.

Proof. The proof of (i) and (iii) is straightforward by using that $f(0)=0$. Property (ii) comes with the following splitting

$S_{k}^{[n]}[f]\left(c_{1}, \ldots, c_{n-1}, 0\right)=\sum_{\substack{\boldsymbol{i} \in I_{k}^{[n]} \\ n \in \boldsymbol{i}}} f\left(c_{i_{1}}+c_{i_{2}}+\cdots+c_{i_{k}}\right)+\sum_{\substack{\boldsymbol{i} \in I_{k}^{[n]} \\ n \notin \boldsymbol{i}}} f\left(c_{i_{1}}+c_{i_{2}}+\cdots+c_{i_{k}}\right)$.

In the first sum, $n$ belongs to any $k$-uplet $\boldsymbol{i}$; it means that $i_{k}=n$. Since $c_{n}=0$, the first sum is exactly $S_{k-1}^{[n-1]}[f]\left(c_{1}, \ldots, c_{n-1}\right)$. In the second sum, $n$ does not belong to the $k$-uplet $i$; it means that the summation is performed over all the $k$-uplets of $(0, \ldots, n-1)$. The second sum is exactly $S_{k}^{[n-1]}[f]\left(c_{1}, \ldots, c_{n-1}\right)$.

With these properties at hand, we can now prove Proposition 3.1.

Proof (of proposition 3.1). Let $1 \leqslant k<n$. Using (i) and (ii) of Lemma C.1, we find from the equality (3.2) that

$$
\begin{aligned}
\Psi_{k}^{[n]}[f]\left(c_{1}, \ldots, c_{n-1}, 0\right)= & \sum_{2 \leqslant s \leqslant k}(-1)^{k-s}\left(\begin{array}{c}
n-s \\
k-s
\end{array}\right) S_{s-1}^{[n-1]}[f]\left(c_{1}, \ldots, c_{n-1}\right) \\
& +\sum_{1 \leqslant s \leqslant k}(-1)^{k-s}\left(\begin{array}{c}
n-s \\
k-s
\end{array}\right) S_{s}^{[n-1]}[f]\left(c_{1}, \ldots, c_{n-1}\right) .
\end{aligned}
$$

We renumber the first sum and use the recursive formula for binomial coefficients

$$
\left(\begin{array}{l}
n-s \\
k-s
\end{array}\right)-\left(\begin{array}{c}
n-s-1 \\
k-s-1
\end{array}\right)=\left(\begin{array}{c}
n-s-1 \\
k-s
\end{array}\right), \quad 1 \leqslant s \leqslant k-1
$$

to obtain (i). Property (ii) is obtained in the same way.

\section{Proof of proposition 3.2}

In order to evaluate $L_{n}^{[n]}\left(F^{[n]}\right)$, we proceed step by step: we first evaluate $L_{n}^{[n]}\left(S_{k}^{[n]}[f]\right)$ and then $L_{n}^{[n]}\left(\Psi_{k}^{[n]}[f]\right)$. 
Lemma D.1. For all $1 \leqslant k<n$, we have the following equalities

$$
\text { (i) } \begin{aligned}
L_{n}^{[n]}\left(S_{k}^{[n]}[f]\right)\left(c_{1}, \ldots, c_{n-1}, 0\right)= & \frac{n-k}{n \sigma} S_{k-1}^{[n-1]}\left[f^{\prime}\right]\left(c_{1}, \ldots, c_{n-1}\right) \\
& -\frac{k}{n \sigma} S_{k}^{[n-1]}\left[f^{\prime}\right]\left(c_{1}, \ldots, c_{n-1}\right),
\end{aligned}
$$

(ii) $L_{n}^{[n]}\left(S_{n}^{[n]}[f]\right)\left(c_{1}, \ldots, c_{n-1}, 0\right)=0$.

Proof. We first remark that

$$
\partial_{n} S_{k}^{[n]}[f]\left(c_{1}, \ldots, c_{n-1}, 0\right)=S_{k-1}^{[n-1]}\left[f^{\prime}\right]\left(c_{1}, \ldots, c_{n-1}\right), \quad \forall 1 \leqslant k \leqslant n .
$$

Moreover, following the same reasonning as in the proof of Lemma C.1, we have, for $1 \leqslant s, k<n$

$$
\begin{aligned}
\partial_{s} S_{k}^{[n]}[f]\left(c_{1}, \ldots, c_{n-1}, 0\right) & =\sum_{\substack{\boldsymbol{i} \in I^{[n]} \\
s \in \boldsymbol{i}, n \in \boldsymbol{i}}} f^{\prime}\left(c_{i_{1}}+\cdots+c_{i_{k}}\right)+\sum_{\substack{\boldsymbol{i} \in I_{i}^{[n]} \\
s \in \boldsymbol{i}, n \notin \boldsymbol{i}}} f^{\prime}\left(c_{i_{1}}+\cdots+c_{i_{k}}\right) \\
& =\sum_{\substack{\boldsymbol{i} \in I_{k-1}^{[n-1]} \\
s \in \boldsymbol{i}}} f^{\prime}\left(c_{i_{1}}+\cdots+c_{i_{k-1}}\right)+\sum_{\substack{\boldsymbol{i} \in I_{k}^{[n-1]} \\
s \in \boldsymbol{i}}} f^{\prime}\left(c_{i_{1}}+\cdots+c_{i_{k}}\right) .
\end{aligned}
$$

We sum over $s$ and count the number of times that a $(k-1)$-uplet or $k$-uplet is involved in the sum. We find, for $1 \leqslant k<n$,

$$
\begin{aligned}
\sum_{1 \leqslant s \leqslant n-1} \partial_{s} S_{k}^{[n]} & {[f]\left(c_{1}, \ldots, c_{n-1}, 0\right) } \\
& =(k-1) S_{k-1}^{[n-1]}\left[f^{\prime}\right]\left(c_{1}, \ldots, c_{n-1}\right)+k S_{k}^{[n-1]}\left[f^{\prime}\right]\left(c_{1}, \ldots, c_{n-1}\right) .
\end{aligned}
$$

In the case where $k=n$, the second term in the right hand side does not appear

$$
\sum_{1 \leqslant s \leqslant n-1} \partial_{s} S_{n}^{[n]}[f]\left(c_{1}, \ldots, c_{n-1}, 0\right)=(n-1) S_{n-1}^{[n-1]}\left[f^{\prime}\right]\left(c_{1}, \ldots, c_{n-1}\right)
$$

The conclusion is obtained by combining the equalities (D.1), (D.2) for (i) and the equalities (D.1) $(k=n)$, (D.3) for (ii).

Since the operator $L_{n}^{[n]}$ is linear, it is now straightforward to find $L_{n}^{[n]}\left(\Psi_{k}^{[n]}[f]\right)$ when $c_{n}=0$.

Lemma D.2. For all $1 \leqslant k<n$, we have the following equalities

(i) $L_{n}^{[n]}\left(\Psi_{k}^{[n]}[f]\right)\left(c_{1}, \ldots, c_{n-1}, 0\right)=-\frac{1}{n \sigma} \sum_{1 \leqslant s \leqslant k-1} \beta_{k, s}^{[n]} S_{s}^{[n-1]}\left[f^{\prime}\right]\left(c_{1}, \ldots, c_{n-1}\right)$

$$
-\frac{k}{n \sigma} S_{k}^{[n-1]}\left[f^{\prime}\right]\left(c_{1}, \ldots, c_{n-1}\right),
$$

(ii) $L_{n}^{[n]}\left(\Psi_{n}^{[n]}[f]\right)\left(c_{1}, \ldots, c_{n-1}, 0\right)=-\frac{1}{n \sigma} \sum_{1 \leqslant s \leqslant n-2} \beta_{n, s}^{[n]} S_{s}^{[n-1]}\left[f^{\prime}\right]\left(c_{1}, \ldots, c_{n-1}\right)$

$$
+\frac{n-1}{n \sigma} S_{n-1}^{[n-1]}\left[f^{\prime}\right]\left(c_{1}, \ldots, c_{n-1}\right)
$$


where the coefficients $\beta_{k, s}^{[n]}$ are given in the statement of Propsition 3.2.

Using again the linearity of the operator $L_{n}^{[n]}$, we eventually obtain the claimed expression of $L_{n}^{[n]}\left(F^{[n]}\right)(\mathbf{c})$ when $c_{n}=0$.

\section{References}

1. F. Boyer. Mathematical study of multi-phase flow under shear through order parameter formulation. Asymptot. Anal., 20(2):175-212, 1999.

2. F. Boyer. A theoretical and numerical model for the study of incompressible mixture flows. Comput. Fluids, 31(1):41-68, 2002.

3. F. Boyer and C. Lapuerta. Study of a three component Cahn-Hilliard flow model. M2AN, 40(4):653-687, 2006.

4. F. Boyer, C. Lapuerta, S. Minjeaud, and B. Piar. A local adaptive refinement method with multigrid preconditionning illustrated by multiphase flows simulations. ESAIM Proceedings, 27:15-53, 2009.

5. F. Boyer, C. Lapuerta, S. Minjeaud, B. Piar, and M. Quintard. Cahn-Hilliard / NavierStokes model for the simulation of three-phase flows. Transp. Porous Media, 82(3):463483, 2010.

6. F. Boyer and S. Minjeaud. Numerical schemes for a three component Cahn-Hilliard model. M2AN, 45(4):697-738, 2011.

7. J. W. Cahn and J. E. Hilliard. Free energy of a nonuniform system i. interfacial free energy. J. Chem. Phys., 2:258-267, 1958.

8. J.W. Cahn. On spinodal decomposition. Acta Metall., 9:795-801, 1961.

9. D. de Fontaine. A computer simulation of the evolution of coherent composition variations in solid solutions. PhD thesis, Northwestern University, 1967.

10. C. M. Elliott. The Cahn-Hilliard model for the kinetics of phase separation. In Mathematical models for phase change problems (Óbidos, 1988), volume 88 of Internat. Ser. Numer. Math., pages 35-73. Birkhäuser, Basel, 1989.

11. C. M. Elliott and H. Garcke. On the Cahn-Hilliard equation with degenerate mobility. SIAM J. Math. Anal., 27(2):404-423, 1996.

12. C. M. Elliott and H. Garcke. Diffusional phase transitions in multicomponent systems with a concentration dependent mobility matrix. Phys. D, 109(3-4):242-256, 1997.

13. C.M. Elliott and S. Luckhaus. A generalised diffusion equation for phase separation of a multi-component mixture with interfacial free energy. IMA Preprint Series, \# 887, 1991.

14. D. J. Eyre. Systems of Cahn-Hilliard equations. SIAM J. Appl. Math., 53(6):16861712, 1993.

15. W. J. Gao and J. X. Yin. Systems of Cahn-Hilliard equations with density-dependent transition matrices. Chinese Ann. Math. Ser. A, 20(2):169-176, 1999.

16. H. Garcke. On Cahn-Hilliard systems with elasticity. Proc. Roy. Soc. Edinburgh Sect. A, 133(2):307-331, 2003.

17. H. Garcke. On a Cahn-Hilliard model for phase separation with elastic misfit. Ann. Inst. H. Poincaré Anal. Non Linéaire, 22(2):165-185, 2005.

18. H. Garcke, B. Nestler, and B. Stoth. A multiphase field concept: numerical simulations of moving phase boundaries and multiple junctions. SIAM J. Appl. Math., 60(1):295315,2000 .

19. M. E. Gurtin. Generalized Ginzburg-Landau and Cahn-Hilliard equations based on a microforce balance. Phys. D, 92(3-4):178-192, 1996. 
20. J.J. Hoyt. The continuum theory of nucleation in multicomponent systems. Acta Metall., 38:1405-1412, 1990.

21. D. Jacqmin. Calculation of two-phase Navier-Stokes flows using phase-field modeling. J. Comput. Phys., 155(1):96-127, 1999.

22. D. Jacqmin. Contact-line dynamics of a diffuse fluid interface. J. Fluid Mech., 402:57$88,2000$.

23. J. Kim. Phase-field models for multi-component fluid flows. Commun. Comput. Phys., 12(3):613-661, 2012.

24. J. Kim, K. Kang, and J. Lowengrub. Conservative multigrid methods for ternary Cahn-Hilliard systems. Commun. Math. Sci., 2(1):53-77, 2004.

25. J. Kim and J. Lowengrub. Phase field modeling and simulation of three-phase flows. Interfaces Free Bound., 7(4):435-466, 2005.

26. J.S. Kirkaldy and D.J. Young. Diffusion in the Condensed State. Book (Institute of Metals). Institute of Materials, 1987.

27. J. Lowengrub and L. Truskinovsky. Quasi-incompressible Cahn-Hilliard fluids and topological transitions. R. Soc. Lond. Proc. Ser. A Math. Phys. Eng. Sci., 454(1978):2617-2654, 1998.

28. S. Maier-Paape, B. Stoth, and T. Wanner. Spinodal decomposition for multicomponent Cahn-Hilliard systems. Journal of Statistical Physics, 98(3-4):871-896, 2000.

29. A. Miranville and G. Schimperna. Generalized Cahn-Hilliard equations for multicomponent alloys. Adv. Math. Sci. Appl., 19(1):131-154, 2009.

30. J. E. Morral and J. W. Cahn. Spinodal decomposition in ternary systems. Acta Metall., 19:1037-1045, 1971.

31. A. Novick-Cohen. The Cahn-Hilliard equation. In Handbook of differential equations: evolutionary equations. Vol. IV, Handb. Differ. Equ., pages 201-228. Elsevier/NorthHolland, Amsterdam, 2008.

32. L. Onsager. Reciprocal relations in irreversible processes I. Phys. Rev., 37:405-426, 1931.

33. L. Onsager. Reciprocal relations in irreversible processes II. Phys. Rev., 38:2265-2279, 1931. 